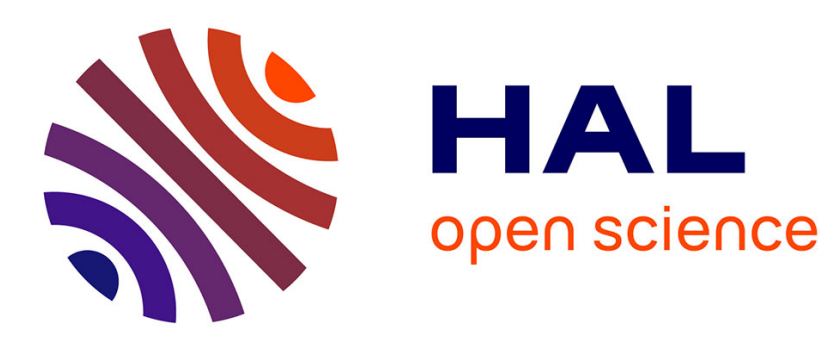

\title{
Global Stabilization of Discrete-Time Integrators System By Bounded Controls
}

Xuefei Yang, Bin Zhou, Frederic Mazenc

\section{To cite this version:}

Xuefei Yang, Bin Zhou, Frederic Mazenc. Global Stabilization of Discrete-Time Integrators System By Bounded Controls. IEEE Transactions on Circuits and Systems I: Regular Papers, 2020. hal03114223

\section{HAL Id: hal-03114223 \\ https://hal.inria.fr/hal-03114223}

Submitted on 18 Jan 2021

HAL is a multi-disciplinary open access archive for the deposit and dissemination of scientific research documents, whether they are published or not. The documents may come from teaching and research institutions in France or abroad, or from public or private research centers.
L'archive ouverte pluridisciplinaire HAL, est destinée au dépôt et à la diffusion de documents scientifiques de niveau recherche, publiés ou non, émanant des établissements d'enseignement et de recherche français ou étrangers, des laboratoires publics ou privés. 


\title{
Global Stabilization of Discrete-Time Integrators System By Bounded Controls
}

\author{
Xuefei Yang, Bin Zhou, Senior Member, IEEE, Frédéric Mazenc
}

\begin{abstract}
This paper is concerned with global stabilization of discretetime multiple integrators with bounded controls by utilizing the energy function based approach. First, a discrete-time double integrators system subject to input additive disturbances is stabilized by a bounded feedback whose formula involves a linear function of the state and a saturation function only. Next, this result is used to stabilize a discrete-time multiple integrators of arbitrary length by bounded control with the aid of a special canonical form. Compared with the existing results, the proposed controllers require fewer saturation functions, which allow a better use of the control energy. Moreover, some free parameters that are introduced into these controllers can help improve the transient performance of the closed-loop systems significantly. Finally, a numerical example is given to illustrate the effectiveness of the proposed method.
\end{abstract}

Keywords: Asymptotic stabilization; discrete-time systems; bounded control; energy function; saturation function.

\section{INTRODUCTION}

A CTUATOR saturation is an inherent phenomenon in practical circuits and systems and thereby many research efforts have been devoted to deal with it in the past decades [5], [6], [7], [8]. In particular, global stabilization of linear systems by bounded feedback has attracted considerable attention. It has been shown that this problem can be solved if and only if the considered system is stabilizable in the ordinary sense and is not exponentially unstable when the control is zero [16], [23]. Such a system is called asymptotically null controllable with bounded controls (ANCBC). Since the work [3] (see also [17]), it is well-known that for a multiple integrators system of dimension $n \geq 3$, which is an ANCBC system, global asymptotic stabilization cannot be achieved by saturated linear state feedback. Therefore, the global stabilization of general ANCBC systems requires the design of more complex control laws.

Multiple integrators systems, also called chains of integrators, have strong potential applications in engineering because they can approximate or be parts of models such as the inertia wheel pendulum and the vertical takeoff and landing aircraft [9], [24], [25], so that their study has a strong engineering motivation and thus attracted considerable attention in the literature [11], [13], [18]. For the global stabilization problem, A. Teel provided a pioneering control strategy by introducing a class of nonlinear controllers consisting of nested saturation functions [18]. This work was later successfully extended to general ANCBC continuous-time and discrete-time linear systems [16], [23]. Unfortunately, for high order systems and/or bigger initial conditions, these nonlinear methods always result in a deteriorated

This work was supported in part by the National Natural Science Foundation of China under grants 61903102, 61773140 and 61773387, and by the National Postdoctoral Program for Innovative Talents under Grant BX20190099. (Corresponding author: Bin Zhou)

Xuefei Yang is with Department of Mechanical Engineering \& Automation, Harbin Institute of Technology(Shenzhen), Shenzhen, Guangdong, 518055, China (e-mail: xfyang1989@163.com, yangxuefei@hit.edu.cn).

Bin Zhou is with the Center for Control Theory and Guidance Technology, Harbin Institute of Technology, Harbin, 150001, China (e-mail: binzhoulee@163.com, binzhou@hit.edu.cn).

Frédéric Mazenc is with EPI DISCO Inria, Laboratoire des Signaux et Systèmes (L2S, UMR CNRS 8506), CentraleSupélec, Université Paris-Sud, 3 rue Joliot Curie, 91192, Gif-sur-Yvette, France (e-mail: frederic.mazenc@l2s.centralesupelec.fr). transient performance. Thereafter, a number of studies have been made to improve the transient performance. For instance, in [10] and [11], state-dependent functions were introduced into the controllers, which have been shown to significantly improve the transient performance of the closed-loop system. Later on, the results in [11] were successfully extended to the discrete-time multiple integrators system [12] and were further improved in [26]. Recently, the global stabilization problem of high order integrators subject to disturbance and saturated control was consider in [1], where an adaptive nested saturation feedback law was proposed. This method was also shown to improve the transient performance of the closed-loop system. When the multiple integrators system is subject to input saturation and time-delay, the corresponding global stabilization problem was for the first time investigated in [13] by extending Teel's forwarding approach [18]. Later on, the same problem was revisited in [29] by using delayed and bounded controls based on some special canonical forms.

In the studies of global stabilization problems mentioned above, two approaches, based respectively on Lyapunov functions and energy functions, are often adopted. When one applies the well-known Lyapunov function based approach, one needs to find a Lyapunov function whose derivative along the trajectories of the studied systems is negative definite [12], [14], [20], [30]. There is no request of this type in case of energy function based analyses: the energy function only has to eventually decrease to zero and can increase on some finite time intervals [18], [28]. In general, the energy function based approach leads to more difficult analyses than the Lyapunov function based approach. That is probably the reason why the energy function based approach has received little attention, with the notable exceptions: [2], [27], [28]. Let us observe that corresponding results for linear discrete-time systems by bounded control, as far as we know, have never been established.

In this paper, we investigate the problem of global stabilization of discrete-time integrators system with bounded controls and adopt an energy function based approach. The contribution of this paper and the significance of the obtained results can be summarized as follows. First, the global stabilization problem for a double integrators subject to input additive disturbances by bounded controls is addressed. Through an energy function based approach, we design a parameterized state feedback and give explicit conditions guaranteeing that all trajectories of the closed-loop system converge to a small neighborhood of the origin in finite steps and remain inside forever. We wish to emphasize that, by contrast with the analysis in [28], the proof for this result is far from trivial. We did our best to propose a simple proof but, due to the high difficulty of the problem, we did not obtain a short proof. Second, based on a new special canonical form (which is different from that used in [12] and [30]) the global stabilization problem of discrete-time multiple integrators with bounded controls is solved via a class of nonlinear control laws in which there are $\left[\frac{n+1}{2}\right]$ nested saturation functions only. In the control laws proposed in [12] and [30] there are $n$ saturation functions so that the designed control laws have significantly less saturations. This is an advantage because this leads to better transient performances. Notice also that we introduce some free parameters in the formula of the 
feedback to make it possible to improve the transient performances further.

Notation. The notation used in this paper is standard. For two integers $p$ and $q$ with $p \leq q$, the symbol $\mathbf{I}[p, q]$ refers to the set $\{p, p+1, \ldots, q\}$. By $I_{m}$, we denote the identity matrix of dimension $n$. For a positive constant $\varepsilon, \sigma_{\varepsilon}(x)=\varepsilon \operatorname{sign}(x) \min \{|x / \varepsilon|, 1\}$ denotes the standard saturation function. Moreover, we will denote $\sigma_{1}(x)$ by $\sigma(x)$ to simplify. The notation $|\cdot|$ refers to both the induced matrix 2 norm and the usual Euclidean vector norm. For a real number $\alpha,[\alpha]$ refers to the integer part of $\alpha$. For a pair of matrices $(A, b)$ with $A \in \mathbf{R}^{n \times n}$ and $b \in \mathbf{R}^{n \times 1}, Q_{\mathrm{c}}(A, b)=\left[b, A b, \ldots, A^{n-1} b\right]$ denotes the controllability matrix of $(A, b)$. We denote by $P>0$ a real symmetric positive definite matrix.

\section{Problem Formulation And Technical Preliminaries}

Throughout the paper, we reconsider the following discrete-time integrators system [12], [30]

$$
x(k+1)=A_{\circ} x(k)+b_{\circ} u(k),
$$

with

$$
A_{\mathrm{o}}=\left[\begin{array}{ccccc}
1 & 1 & 0 & \cdots & 0 \\
& 1 & \ddots & \ddots & \vdots \\
& & \ddots & 1 & 0 \\
& & & 1 & 1 \\
& & & & 1
\end{array}\right], b_{\mathrm{o}}=\left[\begin{array}{c}
0 \\
0 \\
\vdots \\
0 \\
1
\end{array}\right]
$$

where $x=\left[x_{1}, x_{2}, \ldots, x_{n}\right]^{\mathrm{T}} \in \mathbf{R}^{n}$ and $u \in \mathbf{R}$ are state and control variables, respectively. The main task of this paper is to solve the following problem:

Problem 1: Constructing a state feedback $u_{f}$ such that $\left|u_{f}\right| \leq 1$ for all $k \in \mathbb{N}$ and the system (1) in closed-loop with $u_{f}$ is globally asymptotically and locally exponentially stable.

Problem 1 has been investigated by Marchand et al. in [12], in which a bounded nonlinear control law consisting of $n$ saturation functions is proposed based on a special state representation (see Lemma 1 in [12]). To ensure stability, the constraints on the corresponding parameters (see Theorem 1 in [12]) may be conservative and may degrade the control performance. Recently, to solve Problem 1 , we proposed in [30] some new classes of control laws by taking advantage of the state space representation of [12]. The expressions of these control laws have $n$ saturation functions, and the new constrains on the corresponding parameters are less conservative than those in [12]. However, all the nonlinear controllers proposed in [12] and [30] require $n$ saturation functions, which suggests that the actuator capacity is not fully utilized. Bearing this in mind, by virtue of a novel special state representation and a new crucial technique, we construct in the present paper a new family of control laws for solving Problem 1. These controllers only have $\left[\frac{n+1}{2}\right]$ saturation functions and they have some free parameters which are degrees of freedom and can be used to improve the transient performance, as illustrated by a numerical example in Section V.

We end this section with two technical lemmas borrowed from [28], which will play important roles in the proof of our model transformation.

Lemma 1: Let $\left(A_{i}, b_{i}\right), i=1,2$, be two pairs of matrices and let $\left(A_{1}, b_{1}\right)$ be controllable. Then there exists an invertible matrix $T$ such that $A_{2}=T A_{1} T^{-1}, b_{2}=T b_{1}$, if and only if $\left(A_{2}, b_{2}\right)$ is controllable and $\lambda\left(A_{2}\right)=\lambda\left(A_{1}\right)$. In this case, the unique transformation matrix $T$ is given by $T=Q_{\mathrm{c}}\left(A_{2}, b_{2}\right) Q_{\mathrm{c}}^{-1}\left(A_{1}, b_{1}\right)$.
Lemma 2: Consider the matrices:

$$
A_{\mathrm{T}}=\left[\begin{array}{ccccc}
A_{1} & b_{1} f_{2} & \cdots & b_{1} f_{p-1} & b_{1} f_{p} \\
& A_{2} & \cdots & b_{2} f_{p-1} & b_{2} f_{p} \\
& & \ddots & \vdots & \vdots \\
& & & A_{p-1} & b_{p-1} f_{p} \\
& & & & A_{p}
\end{array}\right], b_{\mathrm{T}}=\left[\begin{array}{c}
b_{1} \\
b_{2} \\
\vdots \\
b_{p-1} \\
b_{p}
\end{array}\right] \text {, }
$$

where $b_{i} \in \mathbf{R}^{n_{i} \times 1}, A_{i} \in \mathbf{R}^{n_{i} \times n_{i}}, f_{i} \in \mathbf{R}^{1 \times n_{i}}$ are arbitrary matrices and $n_{1}+n_{2}+\cdots+n_{p}=n$. If $\lambda\left(A_{i}-b_{i} f_{i}\right) \cap \lambda\left(A_{j}\right)=\emptyset, j \in$ $\mathbf{I}[1, i-1], i \in \mathbf{I}[2, p]$, then $\left(A_{\mathrm{T}}, b_{\mathrm{T}}\right)$ is controllable if and only if all the pairs $\left(A_{i}, b_{i}\right), i \in \mathbf{I}[1, p]$, are controllable.

\section{THE Key REsUlt}

In this section, we address Problem 1 when $n=2$. The result will play a crucial role in the next section. To simplify the notation, let

$$
A=\left[\begin{array}{ll}
1 & 1 \\
0 & 1
\end{array}\right] \text { and } b=\left[\begin{array}{l}
0 \\
1
\end{array}\right] \text {. }
$$

Proposition 1: Consider the discrete-time system

$$
\left\{\begin{aligned}
x(k+1) & =A x(k)+b u(k), \quad x \in \mathbf{R}^{2}, \\
u(k) & =-\sigma_{\varepsilon_{2}}\left(F_{\gamma} x(k)+\sigma_{\varepsilon_{1}}(\varpi(k))\right),
\end{aligned}\right.
$$

where $\varepsilon_{1} \geq 0, \varepsilon_{2}>0$ are given scalars, $\varpi(k)$ is an external signal,

$$
F_{\gamma}=\left(1+b^{\mathrm{T}} P b\right)^{-1} b^{\mathrm{T}} P A=\left[\begin{array}{ll}
\gamma^{2} & 2 \gamma
\end{array}\right],
$$

in which $\gamma \in(0,1 / 2]$ and

$$
P=\left[\begin{array}{cc}
\frac{\gamma^{3}}{(\gamma-1)^{2}} & \frac{\gamma^{2}}{(\gamma-1)^{2}} \\
\frac{\gamma^{2}}{(\gamma-1)^{2}} & \frac{1}{(\gamma-1)^{2}}-1
\end{array}\right],
$$

satisfies the discrete-time parametric Lyapunov equation (DPLE):

$$
A^{\mathrm{T}} P A-A^{\mathrm{T}} P b\left(1+b^{\mathrm{T}} P b\right)^{-1} b^{\mathrm{T}} P A=(1-\gamma) P .
$$

If

$$
\left(\sqrt{\frac{2-\gamma}{1-\gamma}}+1\right) \varepsilon_{1}<\varepsilon_{2},
$$

then there exists an integer $k^{*}>0$ such that

$$
\left|F_{\gamma} x(k)+\sigma_{\varepsilon_{1}}(\varpi(k))\right| \leq \varepsilon_{2}, k \geq k^{*} .
$$

and then there holds $x(k+1)=\left(A-b F_{\gamma}\right) x(k)-b \sigma_{\varepsilon_{1}}(\varpi(k))$, $\forall k \geq k^{*}$.

The proof of this proposition is length and will be given in Section VI where we will use a new energy function based method.

Remark 1: For continuous time systems, there is a similar result in [28]. However, by contrast with the analysis in [28], the proof here is far from trivial. For instance, the state-space is first partitioned into three parts $\left(\mathbf{I}^{+}, \mathbf{I I}\right.$ and $\left.\mathbf{I}^{-}\right)$in both the continuous case (see (38) in [28]) and the discrete case (see (30) in Section VI). In the continuous case, one can show that any trajectory starting at region $\mathbf{I}^{+}\left(\mathbf{I}^{-}\right)$will return to $\mathbf{I I}$ in finite time. However, in the discrete case, after finite steps, the trajectory starting in region $\mathbf{I}^{+}\left(\mathbf{I}^{-}\right)$will enter region $\mathbf{I I}$ directly, or jump over $\mathbf{I I}$ and enter region $\mathbf{I}^{-}\left(\mathbf{I}^{+}\right)$ due to the discontinuity. This difficulty is inherent in the trajectory analysis. In addition, There exist many other cases, which are more challenging than those in [28] and need to be treated carefully, as shown in Section VI.

The following corollary is a direct consequence of Proposition 1:

Corollary 1: Consider the system

$$
x(k+1)=A x(k)+b \sigma_{\varepsilon}(u(k)), x \in \mathbf{R}^{2},
$$

where $(A, b)$ is given by (3) and $\varepsilon$ is a positive real number. Then for any $\gamma \in(0,1 / 2]$, the control law 


$$
u(k)=-F_{\gamma} x(k),
$$

where $F_{\gamma}$ is given by (5), globally asymptotically stabilizes system (10).

When the input additive disturbance vanishes, a discrete-time double integrators with bounded controls described by

$$
x(k+1)=A x(k)+b \sigma(u(k)), \quad x \in \mathbf{R}^{2},
$$

where

$$
u(k)=f_{1} x_{1}(k)+f_{2} x_{2}(k),
$$

has been discussed in detail by Yang et al. in [21] and [22]. Different from the continuous time case that any linear state feedback control law, which locally stabilizes the double integrators, also globally stabilizes the double integrators when the control is bounded [15], [19], Yang et al. in [22] has pointed out that, the discrete-time double integrators has intrinsically different behavior, namely, even it is stabilized locally by some linear feedback, it can not be stabilized globally by the same controller when the control is bounded. Later, Yang et al. in [21] further studied the global stabilization of the discrete-time double integrator (12), and proved that the closed-loop system consisting of (12) and (13) is globally asymptotically stable if and only if

$$
\frac{1}{2} f_{1}-2<f_{2}<\frac{3}{2} f_{1}<0 .
$$

Now, let us return to Corollary 1. Let $y(k)=\left[y_{1}(k), y_{2}(k)\right]^{\mathrm{T}}=$ $x(k) / \varepsilon_{2}, v(k)=u(k) / \varepsilon_{2}, \hat{f}_{1}=\gamma^{2}$ and $\hat{f}_{2}=2 \gamma$. Then system (10) and (11) can be rewritten as

$$
\left\{\begin{aligned}
y(k+1) & =A y(k)+b \sigma(v(k)), \quad y \in \mathbf{R}^{2}, \\
v(k) & =-\hat{f}_{1} y_{1}(k)-\hat{f}_{2} y_{2}(k),
\end{aligned}\right.
$$

which is in the form (12)-(13) by setting $f_{1}=-\hat{f}_{1}=-\gamma^{2}$ and $f_{2}=-\hat{f}_{2}=-2 \gamma$. Since $\gamma \in(0,1 / 2]$, the constants $f_{1}=-\gamma^{2}$ and $f_{2}=-2 \gamma$ satisfy (14). Thus, by using the results in [21], we conclude that system (15) (and also the closed-loop system consisting of (10) and (11)) is globally asymptotically stable, which is consistent with Corollary 1.

\section{The Solution to Problem 1}

In this section, with the aid of Proposition 1, a class of nonlinear control laws will be constructed to solve Problem 1. To this end, we need a special state space description of system (1), as stated in the following lemma. For convenience, we let $p=\left[\frac{n+1}{2}\right]$,

$$
\kappa=\left\{\begin{array} { l l } 
{ I _ { 2 } , } & { n \text { is even, } } \\
{ [ 0 , 1 ] , } & { n \text { is odd, } }
\end{array} \quad y _ { 1 } ( k ) \in \left\{\begin{array}{ll}
\mathbf{R}^{2}, & n \text { is even, } \\
\mathbf{R}, & n \text { is odd, }
\end{array}\right.\right.
$$

and

$$
y(k)=\left[\begin{array}{llll}
y_{1}^{\mathrm{T}}(k) & y_{2}^{\mathrm{T}}(k) & \cdots & y_{p}^{\mathrm{T}}(k)
\end{array}\right]^{\mathrm{T}},
$$

where $y_{i}(k) \in \mathbf{R}^{2}, i \in \mathbf{I}[2, p]$.

Lemma 3: Let $\gamma_{i}, i \in \mathbf{I}[2, p]$, be given positive constants. Consider the discrete-time system:

$$
y(k+1)=A_{p} y(k)+b_{p} u(k),
$$

where

$A_{p}=\left[\begin{array}{ccccc}\kappa A \kappa^{\mathrm{T}} & \kappa A_{\gamma_{2}} & \cdots & \kappa A_{\gamma_{p-1}} & \kappa A_{\gamma_{p}} \\ & A & \cdots & A_{\gamma_{p-1}} & A_{\gamma_{p}} \\ & & \ddots & \vdots & \vdots \\ & & & A & A_{\gamma_{p}}\end{array}\right], b_{p}=\left[\begin{array}{c}\kappa b \\ b \\ \vdots \\ b \\ b\end{array}\right]$, in which $(A, b)$ is given by (3), $\kappa$ is given by (16), $y(k)$ is given by (17), and

$$
A_{\gamma_{i}}=\left[\begin{array}{cc}
0 & 0 \\
\gamma_{i}^{2} & 2 \gamma_{i}
\end{array}\right] \triangleq b F_{\gamma_{i}}, i \in \mathbf{I}[1, p] .
$$

Let $T=Q_{\mathrm{c}}\left(A_{p}, b_{p}\right) Q_{\mathrm{c}}^{-1}\left(A_{\mathrm{o}}, b_{\mathrm{o}}\right)$, where $\left(A_{\mathrm{o}}, b_{\mathrm{o}}\right)$ is given by (2) and $\left(A_{p}, b_{p}\right)$ is given by (19). Then system (1) is transformed into (18) by the invertible transformation $y(k)=T x(k)$.

Proof: By Lemma 1, we only need to show that $\left(A_{p}, b_{p}\right)$ is controllable since $\lambda\left(A_{\circ}\right)=\lambda\left(A_{p}\right)$. Let us introduce the function $\mathrm{d}_{i}(\lambda)=\left|\lambda I_{2}-\left(A-b F_{\gamma_{i}}\right)\right|, i \in \mathbf{I}[2, p]$. Then we have $\mathrm{d}_{i}(1)=\gamma_{i}^{2} \neq 0, i \in \mathbf{I}[2, p]$, which implies that $\lambda\left(A-b F_{\gamma_{i}}\right) \cap$ $\left(\lambda\left(\kappa A \kappa^{\mathrm{T}}\right) \cup \lambda(A)\right)=\emptyset, i \in \mathbf{I}[2, p]$. On the other hand, it is easy to verify that both $\left(\kappa A \kappa^{\mathrm{T}}, \kappa b\right)$ and $(A, b)$ are controllable. Therefore, by using Lemma 2 , we conclude that $\left(A_{p}, b_{p}\right)$ is controllable. This concludes the proof.

From Lemma 3 it follows that, if system (18) is globally stabilized by a controller $u(k)=u(y(k))$, then system (1) is also globally stabilized by $u\left(T^{-1} x(k)\right)$. Thus, we only need to design stabilizing controller $u(y(k))$ for the system (18). By virtue of 1 , we are ready to give the main result in this section.

Theorem 1: Let $\gamma_{i}, i \in \mathbf{I}[1, p]$, be given positive constants satisfying $\gamma_{i} \in(0,1 / 2]$. Then Problem 1 is solved by the nonlinear controller $u(k)=-u_{p}(k)$, where

$$
\left\{\begin{aligned}
u_{i}(k) & =\sigma_{\varepsilon_{i}}\left(F_{\gamma_{i}} y_{i}(k)+u_{i-1}(k)\right), i \in \mathbf{I}[2, p], \\
u_{1}(k) & =\sigma_{\varepsilon_{1}}\left(F_{\gamma_{1}} \kappa^{\mathrm{T}} y_{1}(k)\right),
\end{aligned}\right.
$$

in which $y_{i}(k), i \in \mathbf{I}[1, p]$, are defined in Lemma $3, \kappa$ is given by (16), $F_{\gamma_{i}}=\left[\gamma_{i}^{2}, 2 \gamma_{i}\right], i \in \mathbf{I}[1, p]$, are given by (20), and $\varepsilon_{i}, i \in$ $\mathbf{I}[1, p]$, are a series of positive scalars satisfying

$$
\left(\sqrt{\frac{2-\gamma_{i}}{1-\gamma_{i}}}+1\right) \varepsilon_{i-1}<\varepsilon_{i}, i \in \mathbf{I}[2, p], \quad \varepsilon_{p} \leq 1 .
$$

Remark 2: Compared to the results in [12] and [30], the most significant advantage of the control law in Theorem 1 is that it needs less saturation functions, which implies a better use of the control capacity and can lead to better transient performances. This fact will be illustrated by a numerical example in Section V.

Proof: Let us observe from (21) that $\left|u_{i}(k)\right| \leq \varepsilon_{i}, i \in \mathbf{I}[1, p]$. Now, we consider the $p$-th subsystem of the closed-loop system consisting of (18) and (21), namely,

$$
y_{p}(k+1)=A y_{p}(k)-b \sigma_{\varepsilon_{p}}\left(F_{\gamma_{p}} y_{p}(k)+u_{p-1}(k)\right) .
$$

Notice that system (23) is in the form of (4). Then, we deduce from Proposition 1 that if $\left(\sqrt{\left(2-\gamma_{p}\right) /\left(1-\gamma_{p}\right)}+1\right) \varepsilon_{p-1}<\varepsilon_{p}$, namely, (22) is satisfied with $i=p$, there exists a finite integer $k_{p} \geq 0$ such that for all $k \geq k_{p}$, the inequality $\left|F_{\gamma_{p}} y_{p}+u_{p-1}\right| \leq \varepsilon_{p}$ holds. Consequently, $u_{p}(k)=F_{\gamma_{p}} y_{p}(k)+u_{p-1}(k), \forall k \geq k_{p}$. It follows that for all $k \geq k_{p}$, system (18) can be written as

$$
\left\{\begin{aligned}
y_{1}(k+1) & =\kappa A \kappa^{\mathrm{T}} y_{1}(k)+\kappa \sum_{i=2}^{p-1} A_{\gamma_{i}} y_{i}(k)-\kappa b u_{p-1}(k), \\
y_{2}(k+1) & =A y_{2}(k)+\sum_{i=3}^{p-1} A_{\gamma_{i}} y_{i}(k)-b u_{p-1}(k), \\
& \vdots \\
y_{p-1}(k+1) & =A y_{p-1}(k)-b u_{p-1}(k), \\
y_{p}(k+1) & =\left(A-A_{\gamma_{p}}\right) y_{p}(k)-b u_{p-1}(k),
\end{aligned}\right.
$$

We next consider the $y_{p-1}$-system of (24), which is also in the form of (23). Then, arguing as we did above, we deduce that if $\left(\sqrt{\left(2-\gamma_{p-1}\right) /\left(1-\gamma_{p-1}\right)}+1\right) \varepsilon_{p-2}<\varepsilon_{p-1}$, namely, (22) is satisfied with $i=p-1$, there exists an integer $k_{p-1} \geq k_{p}$ such that for all $k \geq k_{p-1}, u_{p-1}(k)=F_{\gamma_{p-1}} y_{p-1}(k)+u_{p-2}(k)$. The 
closed-loop system can be simplified accordingly. By repeating the above process for $y_{i}, i=p-2, p-3, \ldots, 2$, we arrive at the $y_{1}$ subsystem

$$
y_{1}(k+1)= \begin{cases}A y_{1}(k)-b \sigma_{\varepsilon_{1}}\left(F_{\gamma_{1}} y_{1}(k)\right), & n \text { is even } \\ y_{1}(k)-\sigma_{\varepsilon_{1}}\left(2 \gamma_{1} y_{1}(k)\right), & n \text { is odd }\end{cases}
$$

If $n$ is even, system (25) is in the form of (23) where the term $u_{p-1}(k)$ vanishes. Thus, since $0<\varepsilon_{1}$, there exists an integer $k_{1} \geq$ $k_{2}$ such that $y_{1}(k+1)=\left(A-A_{\gamma_{1}}\right) y_{1}(k), \forall k \geq k_{1}$. If $n$ is odd, since $0<\varepsilon_{1}$, we deduce from Lemma 1 in [30] that there exists an integer $k_{1} \geq k_{2}$ such that $y_{1}(k+1)=\left(1-2 \gamma_{1}\right) y_{1}(k), \forall k \geq k_{1}$. To summarize, we have, for all $k \geq k_{1}$,

$$
\left\{\begin{aligned}
y_{1}(k+1)= & \left(\kappa A \kappa^{\mathrm{T}}-\kappa A_{\gamma_{1}} \kappa^{\mathrm{T}}\right) y_{1}(k), \\
y_{2}(k+1)= & \left(A-A_{\gamma_{2}}\right) y_{2}(k)-A_{\gamma_{1}} \kappa^{\mathrm{T}} y_{1}(k), \\
\vdots & \\
y_{p}(k+1)= & \left(A-A_{\gamma_{p}}\right) y_{p}(k)-\sum_{i=2}^{p-1} A_{\gamma_{i}} y_{i}(k) \\
& -A_{\gamma_{1}} \kappa^{\mathrm{T}} y_{1}(k) .
\end{aligned}\right.
$$

Thus the closed-loop system is asymptotically stable if and only if the following series of systems

$$
\left\{\begin{array}{l}
\varphi_{1}(k+1)= \begin{cases}\left(A-A_{\gamma_{1}}\right) \varphi_{1}(k), & n \text { is even } \\
\left(1-2 \gamma_{1}\right) \varphi_{1}(k), & n \text { is odd }\end{cases} \\
\varphi_{i}(k+1)=\left(A-A_{\gamma_{i}}\right) \varphi_{i}(k), i \in \mathbf{I}[2, p],
\end{array}\right.
$$

are all asymptotically stable, which is obvious since $\gamma_{i} \in(0,1 / 2]$ for all $i \in \mathbf{I}[1, p]$. This concludes the proof.

\section{A NumericAl EXAMPLE}

We illustrate the main result of our work with system (1) when $n=4$.

- Control Law 1: This control law is based on Theorem 1 and is given by

$$
u_{1}=-\sigma_{\varepsilon_{2}}\left(F_{\gamma_{2}} y_{2}+\sigma_{\varepsilon_{1}}\left(F_{\gamma_{1}} y_{1}\right)\right) \text {, }
$$

where $F_{\gamma_{i}}=\left[\gamma_{i}^{2}, 2 \gamma_{i}\right], \gamma_{i} \in(0,1 / 2], i \in \mathbf{I}[1,2], y_{i} \in$ $\mathbf{R}^{2}, i \in \mathbf{I}[1,2]$,

$$
y=\left[\begin{array}{l}
y_{1} \\
y_{2}
\end{array}\right]=\left[\begin{array}{cccc}
\gamma_{2}^{2} & 2 \gamma_{2} & 1 & 0 \\
0 & \gamma_{2}^{2} & 2 \gamma_{2} & 1 \\
0 & 0 & 1 & 0 \\
0 & 0 & 0 & 1
\end{array}\right] x
$$

and $\varepsilon_{2}=1, \varepsilon_{1}=\eta\left(\sqrt{\left(1-\gamma_{2}\right)\left(2-\gamma_{2}\right)}-\left(1-\gamma_{2}\right)\right) \varepsilon_{2}, \eta<$ 1 . For the simulation purpose, we choose $\eta=0.99$.

- Control Law 2: This control law is given by [30] and takes the form

$$
u_{4}=-\sigma_{\varepsilon_{4}}\left(\lambda_{4} y_{4}+\sigma_{\varepsilon_{3}}\left(\lambda_{3} y_{3}+\sigma_{\varepsilon_{2}}\left(\lambda_{2} y_{2}+\sigma_{\varepsilon_{1}}\left(\lambda_{1} y_{1}\right)\right)\right)\right),
$$

where the variables $y_{i} \in \mathbf{R}, i \in \mathbf{I}[1,4]$, are given by

$$
y=\left[\begin{array}{l}
y_{1} \\
y_{2} \\
y_{3} \\
y_{4}
\end{array}\right]=\left[\begin{array}{cccc}
t_{11} & t_{12} & t_{13} & 1 \\
0 & t_{22} & t_{23} & 1 \\
0 & 0 & t_{33} & 1 \\
0 & 0 & 0 & 1
\end{array}\right] x,
$$

with $t_{11}=\lambda_{2} \lambda_{3} \lambda_{4}, t_{12}=\lambda_{2} \lambda_{3}+\lambda_{2} \lambda_{4}+\lambda_{3} \lambda_{4}, t_{13}=\lambda_{2}+\lambda_{3}+$ $\lambda_{4}, t_{22}=\lambda_{3} \lambda_{4}, t_{23}=\lambda_{3}+\lambda_{4}$ and $t_{33}=\lambda_{4}, \varepsilon_{i}, i \in \mathbf{I}[1,4]$, satisfy $\varepsilon_{i-1}<\varepsilon_{i} / 2, i \in \mathbf{I}[2,4], \varepsilon_{4} \leq 1$, and $\lambda_{i}, i \in \mathbf{I}[1,4]$, satisfy $0<\lambda_{i}<1, i \in \mathbf{I}[1,4]$. Let $\eta$ be a prescribed number and choose $\varepsilon_{4}=1, \varepsilon_{3}=\eta / 2, \varepsilon_{2}=\eta^{2} / 4, \varepsilon_{1}=\eta^{3} / 8, \eta<1$. For the simulation purpose, we choose $\eta=0.99$.
- Control Law 3: This control law is given in [12] and takes the form

$$
u_{5}=-\delta \sum_{i=1}^{4} \lambda_{i} \sigma_{M_{i}}\left(\frac{y_{i}}{\delta}\right)
$$

where $y_{i}, i \in \mathbf{I}[1,4]$, are given by (28), and $M_{4}=1, M_{j}=1+$ $\alpha_{j}\left(M_{j+1}-\left|\sigma_{M_{j+1}}\left(y_{j+1} / \delta\right)\right|\right) \lambda_{j+1} / \lambda_{j}, j \in \mathbf{I}[1,3]$, in which $\alpha_{i} \in[0,1], i \in \mathbf{I}[1,3]$, and $\delta=1 /\left(\lambda_{1}+\lambda_{2}+\lambda_{3}+\lambda_{4}\right)$ with $0<\Sigma_{i=1}^{k} \lambda_{i}<\lambda_{k}<1, k \in \mathbf{I}[2,4]$. In order to achieve the fastest convergence of the states, we set $\alpha_{i}=1, i \in \mathbf{I}[1,3]$, and $\lambda_{i}=0.5437^{4-i+1}, i \in \mathbf{I}[1,4]$.

We first illustrate how to choose the parameters $\gamma_{i}, i \in \mathbf{I}[1,2]$, in the controller (26). As in the analysis in [30], to achieve a satisfactory transient performance, a trial-and-error procedure via numerical simulation is necessary. That way one determines suitable parameters $\gamma_{i}, i \in \mathbf{I}[1,2]$, for the controller (26). To this end, we let $\gamma_{i}=\gamma, i \in \mathbf{I}[1,2]$. For a given initial condition $x(0)=[-5,-5,5,5]^{\mathrm{T}}$, the trajectories of the closed-loop system under Control Law 1 with different $\gamma$ are plotted in Fig. 1. It follows that, for Control Law 1, the parameter $\gamma=0.15$ leads to the best control performance. Let $\lambda_{i}=\lambda, i \in \mathbf{I}[1,4]$, in (27). Similarly, we can also show that for Control Law 2, the parameter $\lambda=0.1$ leads to the best control performance.

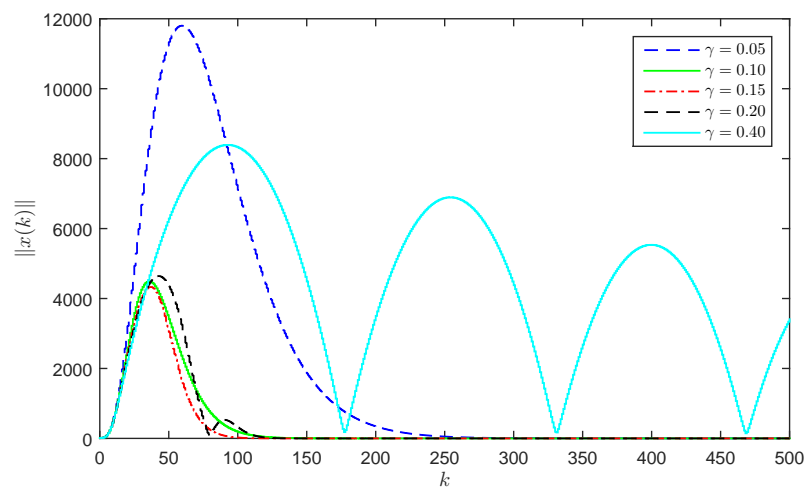

Fig. 1. Trajectories of the 4-th order discrete-time multiple integrators system under Control Law 1 with different $\gamma$.

Finally, we make a comparison of Control Laws 1-3. Let the parameters in Control Laws 1-3 be chosen as the optimal ones obtained above. The resulting 2-norms of the state trajectories of the closed-loop systems are recorded in Fig. 2. From the figure, we clearly see that Control Law 1 established in this paper leads to a much better transient performance than the other ones.

\section{Proof of Proposition 1}

Let the state-space be partitioned as follows:

$$
\left\{\begin{array}{l}
\mathbf{I}^{+}: F_{\gamma} x>\varepsilon_{1}+\varepsilon_{2}, \\
\mathbf{I I}:\left|F_{\gamma} x\right| \leq \varepsilon_{1}+\varepsilon_{2}, \\
\mathbf{I}^{-}: F_{\gamma} x<-\left(\varepsilon_{1}+\varepsilon_{2}\right),
\end{array}\right.
$$

which means that the $x_{1}-x_{2}$ plane is divided into three regions by two lines $F_{\gamma} x= \pm\left(\varepsilon_{1}+\varepsilon_{2}\right)$ (see Fig.3 for an illustration). For future use, let us introduce: $\Omega_{1}=\left\{x=\left[x_{1}, x_{2}\right]^{\mathrm{T}} \mid-\rho_{1} \leq \gamma^{2} x_{2} \leq \rho_{1}\right\} \cap \mathbf{I I}$ and $\Omega_{2}=\left\{x=\left[x_{1}, x_{2}\right]^{\mathrm{T}} \mid V(x) \leq q\right\}$, where $\rho_{1}=2\left(\varepsilon_{1}+\varepsilon_{2}\right)-$ $2 \gamma \varepsilon_{2}+\gamma^{2} \varepsilon_{2}, V(x)=x^{\mathrm{T}} P x$, and $q$ is the positive constant:

$$
q=\mu \varepsilon_{1}^{2},
$$




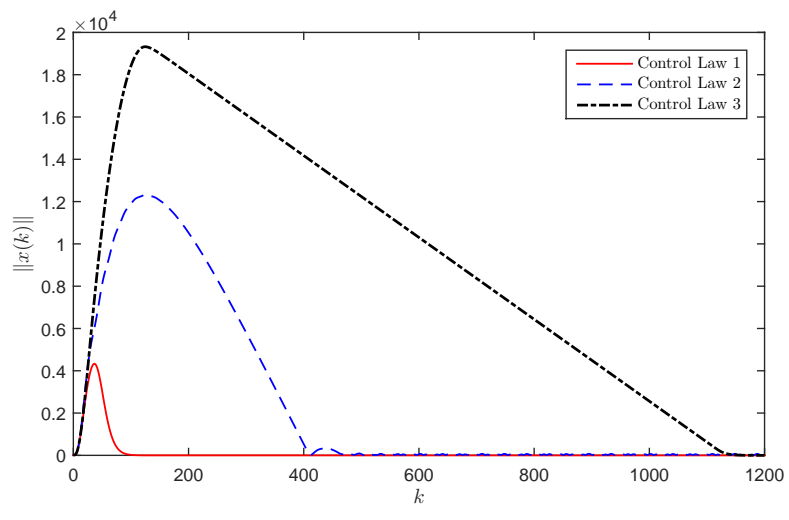

Fig. 2. Comparison of the trajectories of the 4-th order discrete-time multiple integrators system with different controllers.

where $\mu$ is a constant satisfying

$$
\left\{\begin{array}{c}
\frac{1}{\gamma(1-\gamma)^{2}}<\mu<\frac{4(1-\gamma)}{\gamma^{3}(1-\gamma)^{2}} \\
(\sqrt{\gamma(1-\gamma)(2-\gamma) \mu}+1) \varepsilon_{1} \leq \varepsilon_{2} .
\end{array}\right.
$$

The existence of $\mu$ is due to (8).

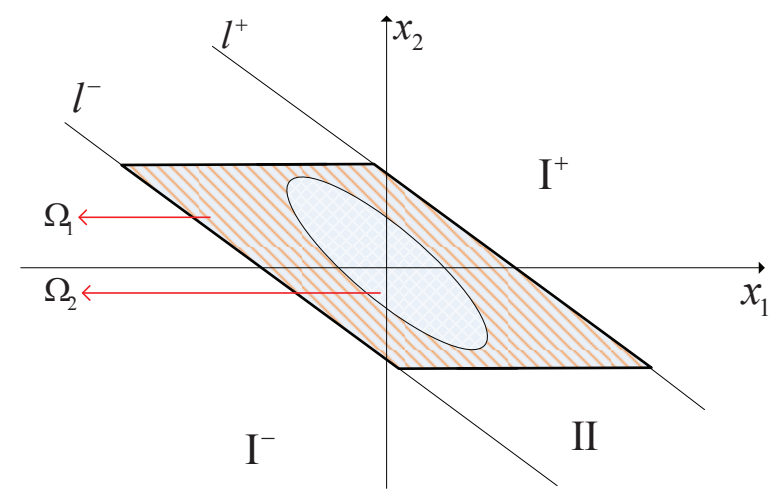

Fig. 3. Partitioning of the state space according to (30). The lines $l^{+}$: $F_{\gamma} x=\varepsilon_{1}+\varepsilon_{2}$, and $l^{-}: F_{\gamma} x=-\left(\varepsilon_{1}+\varepsilon_{2}\right) \cdot \Omega_{1}=\left\{x=\left[x_{1}, x_{2}\right]^{\mathrm{T}}\right.$; $\left.-\rho_{1} \leq \gamma^{2} x_{2} \leq \rho_{1}\right\} \cap \mathbf{I I}, \Omega_{2}=\left\{x=\left[x_{1}, x_{2}\right]^{\mathrm{T}} \mid V(x) \leq q\right\}$.

It turns out that $\Omega_{2} \subset \Omega_{1}$. This fact can be verified as follows: $\Omega_{2} \subset$ II if and only if

$$
\left(\frac{F_{\gamma}}{\varepsilon_{1}+\varepsilon_{2}}\right)\left(\frac{P}{q}\right)^{-1}\left(\frac{F_{\gamma}^{\mathrm{T}}}{\varepsilon_{1}+\varepsilon_{2}}\right)<1 .
$$

Combining (5), (6), (31) and (33) gives $\varepsilon_{2} \quad>$ $(\sqrt{\gamma(1-\gamma)(2-\gamma) \mu}-1) \varepsilon_{1}$, which can be guaranteed by (32) and thus we have $\Omega_{2} \subset$ II. We next show that $\Omega_{2} \subset \Omega_{1}$. To this end, we only need to show that

$$
\gamma^{2} x_{2}=\rho_{1}, x^{\mathrm{T}} P x=q,
$$

has no solution. Substituting (6) and (31) into (34) gives $d_{1} x_{1}^{2}+$ $d_{2} x_{1}+d_{3}=0$, where $d_{1}=\gamma^{3} /(1-\gamma)^{2}, d_{2}=2 \rho_{1} /(1-\gamma)^{2}, d_{3}=$ $(2-\gamma) \rho_{1}^{2} /\left(\gamma^{3}(1-\gamma)^{2}\right)-\mu \varepsilon_{1}^{2}$. Notice that from $(32), \gamma \in(0,1 / 2]$ and $\rho_{1}>2 \varepsilon_{1}$, we have

$$
\begin{aligned}
& d_{2}^{2}-4 d_{1} d_{3} \\
= & -\frac{4}{(1-\gamma)^{3}} \rho_{1}^{2}+\frac{4 \gamma^{3}}{(1-\gamma)^{2}} \mu \varepsilon_{1}^{2}
\end{aligned}
$$

$$
\begin{aligned}
& <-\frac{4}{(1-\gamma)^{3}}\left(2 \varepsilon_{1}\right)^{2}+\frac{4 \gamma^{3}}{(1-\gamma)^{2}} \frac{4(1-\gamma)}{\gamma^{3}(1-\gamma)^{2}} \varepsilon_{1}^{2} \\
& =-\frac{16}{(1-\gamma)^{3}} \varepsilon_{1}^{2}+\frac{16}{(1-\gamma)^{3}} \varepsilon_{1}^{2} \\
& =0
\end{aligned}
$$

which implies that (34) has no solution. Therefore, we have $\Omega_{2} \subset \Omega_{1}$.

Now consider the nonlinear system (4). We have the following claims:

1) For any initial state $x(0) \in \mathbf{I}^{+}$or $\mathbf{I}^{-}$, there exists a finite integer $k>0$ such that $x(k) \in \mathbf{I I}$.

2) For any initial state $x(0) \in \mathbf{I I} \backslash \Omega_{1}$, there exists a finite integer $k>0$ such that $x(k) \in \Omega_{1}$.

3) For any initial state $x(0) \in \Omega_{1} \backslash \Omega_{2}$, if $x(1) \in \mathbf{I I}$, then $x(1) \in$ $\Omega_{1}$ and $V(x(1))-V(x(0))<-v_{1}$ where $v_{1}$ is a suitable positive constant.

4) For any initial state $x(0) \in \Omega_{1} \backslash \Omega_{2}$, if $x(1) \in \mathbf{I}^{+}$or $\mathbf{I}^{-}$, there exists a finite integer $k>1$ such that $x(k) \in \Omega_{1}$ and $V(x(k))-V(x(0))<-v_{2}$ where $v_{2}$ is a suitable positive constant independent of $k$.

5) If there exists an integer $k \geq 0$ such that $x(k) \in \Omega_{2}$, then $\left|F_{\gamma} x(k)+\sigma_{\varepsilon_{1}}(\varpi(k))\right|$

$\leq \varepsilon_{2}$ and $x(k+1) \in \Omega_{2}$.

We conclude from Claims 1-4 that for any initial condition $x(0) \in \mathbf{I}^{+} \cup \mathbf{I}^{-} \cup \mathbf{I I}$, there exists a finite integer $k^{*} \geq 0$ such that $x\left(k^{*}\right) \in \Omega_{2}$. From Claim 5, we know that if the state $x$ enters the bounded set $\Omega_{2}$, it will remain inside forever. Hence, we have $x(k) \in \Omega_{2}, \forall k \geq k^{*}$. Moreover, from Claim 5 we can also get $\left|F_{\gamma} x(k)+\sigma_{\varepsilon_{1}}(\varpi(k))\right| \leq \varepsilon_{2}, \forall k \geq k^{*}$, which implies that $u(k)$ in (4) satisfies $u(k)=-F_{\gamma} x(k)-\sigma_{\varepsilon_{1}}(\varpi), \forall k \geq k^{*}$, which ends the proof of Proposition 1. In the following, we prove Claims 1-5.

Proof of Claim 1

Step 1.1: We show that any trajectory starting in $\mathbf{I}^{+}$will come to $\mathbf{I I} \cup \mathbf{I}^{-}$after finite steps. Notice that in region $\mathbf{I}^{+}, u(k)=$ $-\sigma_{\varepsilon_{2}}\left(F_{\gamma} x(k)+\sigma_{\varepsilon_{1}}(\varpi(k))\right)=-\varepsilon_{2}$, by which the nonlinear system (4) can be expressed as

$$
x(k+1)=A x(k)-b \varepsilon_{2} .
$$

Assume that at the initial time $k=0$, the state $x(0)=$ $\left[x_{1}(0), x_{2}(0)\right]^{\mathrm{T}}$ is in region $\mathbf{I}^{+}$, namely,

$$
F_{\gamma} x(0)=\gamma^{2} x_{1}(0)+2 \gamma x_{2}(0)>\varepsilon_{1}+\varepsilon_{2} .
$$

By solving (35), we obtain

$$
\begin{aligned}
{\left[\begin{array}{l}
x_{1}(k) \\
x_{2}(k)
\end{array}\right] } & =A^{k}\left[\begin{array}{l}
x_{1}(0) \\
x_{2}(0)
\end{array}\right]-\sum_{i=0}^{k-1} A^{i} b \varepsilon_{2} \\
& =\left[\begin{array}{c}
x_{1}(0)+k x_{2}(0)-\frac{k(k-1)}{2} \varepsilon_{2} \\
x_{2}(0)-k \varepsilon_{2}
\end{array}\right] .
\end{aligned}
$$

Now consider the equation $0=F_{\gamma} x(k)-\left(\varepsilon_{1}+\varepsilon_{2}\right)=-\gamma^{2} \varepsilon_{2} k^{2} / 2+$ $\left(\gamma^{2} x_{2}(0)+\gamma^{2} \varepsilon_{2} / 2-2 \gamma \varepsilon_{2}\right) k+\gamma^{2} x_{1}(0)+2 \gamma x_{2}(0)-\left(\varepsilon_{1}+\varepsilon_{2}\right)$, which is quadratic and admits a unique positive solution since $\gamma^{2} x_{1}(0)+2 \gamma x_{2}(0)-\left(\varepsilon_{1}+\varepsilon_{2}\right)>0$ in view of (36). Since $k$ should be a positive integer, it follows that $x$ will enter $\mathbf{I I} \cup \mathbf{I}^{-}$after finite steps. The region $\mathbf{I}^{-}$can be considered by symmetry.

Step 1.2: We show that if the trajectory of $x$ skips back and forth between regions $\mathbf{I}^{+}$and $\mathbf{I}^{-}$(namely, $\mathbf{I}^{+} \rightarrow \mathbf{I}^{-} \rightarrow \mathbf{I}^{+} \rightarrow \mathbf{I}^{-} \rightarrow \cdots$ ), it will enter region II after finite steps. Assume that $x(0) \in \mathbf{I}^{+}$, namely, (36) holds. Without loss of generality, we let $x(1) \in \mathbf{I}^{-}$. Then, by virtue of (37), we get

$$
\begin{aligned}
F_{\gamma} x(1) & =\gamma^{2} x_{1}(1)+2 \gamma x_{2}(1) \\
& =\gamma^{2} x_{1}(0)+2 \gamma x_{2}(0)+\gamma^{2} x_{2}(0)-2 \gamma \varepsilon_{2}
\end{aligned}
$$




$$
<-\left(\varepsilon_{1}+\varepsilon_{2}\right) .
$$

Combining (36) and (38) gives

$$
f_{1}<\gamma^{2} x_{1}(0)+2 \gamma x_{2}(0)<f_{2}
$$

where

$$
f_{1}=\varepsilon_{1}+\varepsilon_{2}, f_{2}=-\left(\varepsilon_{1}+\varepsilon_{2}\right)-\gamma^{2} x_{2}(0)+2 \gamma \varepsilon_{2} .
$$

Therefore,

$$
\gamma^{2} x_{2}(0)<-2\left(\varepsilon_{1}+\varepsilon_{2}\right)+2 \gamma \varepsilon_{2}<0,
$$

where we have noticed that $\gamma \in(0,1 / 2]$. It follows from (41) that if $x$ skips from $\mathbf{I}^{+}$to $\mathbf{I}^{-}$, then $x \in \Omega_{3} \triangleq\left\{x=\left[x_{1}, x_{2}\right]^{\mathrm{T}}\right.$ $\left.\gamma^{2} x_{1}+2 \gamma x_{2}>\varepsilon_{1}+\varepsilon_{2}, \gamma^{2} x_{2}<0\right\}$. In region $\mathbf{I}^{-}$, we have $u(k)=-\sigma_{\varepsilon_{2}}\left(F_{\gamma} x(k)+\sigma_{\varepsilon_{1}}(\varpi(k))\right)=\varepsilon_{2}$. Let us use the notation: $y(k)=x(k+1)$. Then the nonlinear system (4) in $\mathbf{I}^{-}$can be expressed as

$$
\begin{aligned}
y(k) & =A^{k}\left[\begin{array}{l}
y_{1}(0) \\
y_{2}(0)
\end{array}\right]+\sum_{i=0}^{k-1} A^{i} b \varepsilon_{2} \\
& =\left[\begin{array}{c}
x_{1}(0)+(k+1) x_{2}(0)+\frac{k(k-3)}{2} \varepsilon_{2} \\
x_{2}(0)-\varepsilon_{2}+k \varepsilon_{2}
\end{array}\right] .
\end{aligned}
$$

From Step 1.1, we know that $y$ will enter region $\mathbf{I I} \cup \mathbf{I}^{+}$after finite steps. If $y$ enters region $\mathbf{I}^{+}$, there exists an integer $k_{1}>0$ such that

$$
F_{\gamma} y\left(k_{1}\right)<-\left(\varepsilon_{1}+\varepsilon_{2}\right), F_{\gamma} y\left(k_{1}+1\right)>\left(\varepsilon_{1}+\varepsilon_{2}\right),
$$

namely, $f_{3}\left(k_{1}\right)<\gamma^{2} y_{1}\left(k_{1}\right)+2 \gamma y_{2}\left(k_{1}\right)<f_{4}$, where

$$
f_{3}(k)=\left(\varepsilon_{1}+\varepsilon_{2}\right)-\gamma^{2} y_{2}\left(k_{1}\right)-2 \gamma \varepsilon_{2}, f_{4}=-\left(\varepsilon_{1}+\varepsilon_{2}\right) .
$$

Moreover, it follows from (42) and (43) that $f_{5}\left(k_{1}\right)<\gamma^{2} x_{1}(0)+$ $2 \gamma x_{2}(0)<f_{6}\left(k_{1}\right)$, where

$$
\left\{\begin{aligned}
f_{5}\left(k_{1}\right)= & -\frac{1}{2} \gamma^{2} \varepsilon_{2} k_{1}^{2}+\left(\frac{1}{2} \gamma^{2} \varepsilon_{2}-\gamma^{2} x_{2}(0)-2 \gamma \varepsilon_{2}\right) k_{1} \\
& -2 \gamma^{2} x_{2}(0)+\gamma^{2} \varepsilon_{2}+\left(\varepsilon_{1}+\varepsilon_{2}\right) \\
f_{6}\left(k_{1}\right)= & -\frac{1}{2} \gamma^{2} \varepsilon_{2} k_{1}^{2}+\left(\frac{3}{2} \gamma^{2} \varepsilon_{2}-\gamma^{2} x_{2}(0)-2 \gamma \varepsilon_{2}\right) k_{1} \\
& -\gamma^{2} x_{2}(0)+2 \gamma \varepsilon_{2}-\left(\varepsilon_{1}+\varepsilon_{2}\right) .
\end{aligned}\right.
$$

When $y$ skips from $\mathbf{I}^{-}$to $\mathbf{I}^{+}$, we denote $z(k)=y\left(k+k_{1}+1\right)$. Then the equation of state in $\mathbf{I}^{+}$can be expressed as

$$
\begin{aligned}
z(k) & =A^{k}\left[\begin{array}{c}
z_{1}(0) \\
z_{2}(0)
\end{array}\right]-\sum_{i=0}^{k-1} A^{i} b \varepsilon_{2} \\
& =\left[\begin{array}{c}
y_{1}\left(k_{1}\right)+(k+1) y_{2}\left(k_{1}\right)-\frac{k(k-3)}{2} \varepsilon_{2} \\
y_{2}\left(k_{1}\right)+\varepsilon_{2}-k \varepsilon_{2}
\end{array}\right] .
\end{aligned}
$$

From Step 1.1 again we know that $z$ will enter region $\mathbf{I I} \cup \mathbf{I}^{-}$after finite steps. If $z$ enters region $\mathbf{I}^{-}$, there exists an integer $k_{2}>0$ such that

$$
F_{\gamma} z\left(k_{2}\right)>\left(\varepsilon_{1}+\varepsilon_{2}\right), F_{\gamma} z\left(k_{2}+1\right)<-\left(\varepsilon_{1}+\varepsilon_{2}\right) .
$$

It follows from (46) and (47) that $f_{7}\left(k_{2}\right)<\gamma^{2} y_{1}\left(k_{1}\right)+2 \gamma y_{2}\left(k_{1}\right)<$ $f_{8}\left(k_{2}\right)$, where

$$
\left\{\begin{aligned}
f_{7}\left(k_{2}\right)= & \frac{1}{2} \gamma^{2} \varepsilon_{2} k_{2}^{2}+\left(-\frac{3}{2} \gamma^{2} \varepsilon_{2}-\gamma^{2} y_{2}\left(k_{1}\right)+2 \gamma \varepsilon_{2}\right) k_{2} \\
& -\gamma^{2} y_{2}\left(k_{1}\right)-2 \gamma \varepsilon_{2}+\left(\varepsilon_{1}+\varepsilon_{2}\right), \\
f_{8}\left(k_{2}\right)= & \frac{1}{2} \gamma^{2} \varepsilon_{2} k_{2}^{2}+\left(-\frac{1}{2} \gamma^{2} \varepsilon_{2}-\gamma^{2} y_{2}\left(k_{1}\right)+2 \gamma \varepsilon_{2}\right) k_{2} \\
& -2 \gamma^{2} y_{2}\left(k_{1}\right)-\gamma^{2} \varepsilon_{2}-\left(\varepsilon_{1}+\varepsilon_{2}\right) .
\end{aligned}\right.
$$

To conclude, if the trajectory starting at $x(0)=\left[x_{1}(0), x_{2}(0)\right]^{\mathrm{T}} \in$ $\mathrm{I}^{+}$moves along the path $\mathrm{I}^{+} \rightarrow \mathrm{I}^{-} \rightarrow \mathrm{I}^{+} \rightarrow \mathrm{I}^{-}$, there must exist two integers $k_{1}>0$ and $k_{2}>0$ such that

$$
f_{1}<f_{6}\left(k_{1}\right), f_{5}\left(k_{1}\right)<f_{2}, f_{3}<f_{8}\left(k_{2}\right), f_{7}\left(k_{2}\right)<f_{4} .
$$

Denote $f_{9}=-\left(\varepsilon_{1}+\varepsilon_{2}\right)+4 \gamma \varepsilon_{2}-2 \gamma^{2} \varepsilon_{2}, f_{10}\left(k_{1}\right)=f_{5}\left(k_{1}\right)-$ $2\left(\varepsilon_{1}+\varepsilon_{2}\right), f_{11}=\left(\varepsilon_{1}+\varepsilon_{2}\right)-4 \gamma \varepsilon_{2}+2 \gamma^{2} \varepsilon_{2}, f_{12}\left(k_{2}\right)=f_{8}\left(k_{2}\right)+$ $2\left(\varepsilon_{1}+\varepsilon_{2}\right)$. Then it follows from (40), (44), (45) and (48) that

$$
f_{1}>f_{9}, f_{5}\left(k_{1}\right)>f_{10}\left(k_{1}\right), f_{11}>f_{4}, f_{12}\left(k_{2}\right)>f_{8}\left(k_{2}\right) .
$$

Then it follows from (49) and (50) that $k_{1}$ and $k_{2}$ will further satisfy the following inequalities: $f_{9}<f_{6}\left(k_{1}\right), f_{10}\left(k_{1}\right)<f_{2}, f_{3}<$ $f_{12}\left(k_{2}\right), f_{7}\left(k_{2}\right)<f_{11}$, namely,

$$
\left\{\begin{array}{l}
\left(\frac{\gamma^{2} \varepsilon_{2}}{2} k_{1}+\frac{\gamma^{2} \varepsilon_{2}}{2}\right)\left(k_{1}-\left(\frac{-2\left(\gamma^{2} x_{2}(0)+2 \gamma \varepsilon_{2}\right)}{\gamma^{2} \varepsilon_{2}}+4\right)\right)<0, \\
\left(\frac{\gamma^{2} \varepsilon_{2}}{2} k_{1}+\frac{\gamma^{2} \varepsilon_{2}}{2}\right)\left(k_{1}-\left(\frac{-2\left(\gamma^{2} x_{2}(0)+2 \gamma \varepsilon_{2}\right)}{\gamma^{2} \varepsilon_{2}}+2\right)\right)>0, \\
\left(\frac{\gamma^{2} \varepsilon_{2}}{2} k_{2}+\frac{\gamma^{2} \varepsilon_{2}}{2}\right)\left(k_{2}-\left(\frac{2\left(\gamma^{2} y_{2}\left(k_{1}\right)-2 \gamma \varepsilon_{2}\right)}{\gamma^{2} \varepsilon_{2}}+2\right)\right)>0, \\
\left(\frac{\gamma^{2} \varepsilon_{2}}{2} k_{2}+\frac{\gamma^{2} \varepsilon_{2}}{2}\right)\left(k_{2}-\left(\frac{2\left(\gamma^{2} y_{2}\left(k_{1}\right)-2 \gamma \varepsilon_{2}\right)}{\gamma^{2} \varepsilon_{2}}+4\right)\right)<0 .
\end{array}\right.
$$

Solving (51) gives

$$
\left\{\begin{array}{l}
\frac{-2\left(\gamma^{2} x_{2}(0)+2 \gamma \varepsilon_{2}\right)}{\gamma^{2} \varepsilon_{2}}+2<k_{1}<\frac{-2\left(\gamma^{2} x_{2}(0)+2 \gamma \varepsilon_{2}\right)}{\gamma^{2} \varepsilon_{2}}+4 \\
\frac{2\left(\gamma^{2} y_{2}\left(k_{1}\right)-2 \gamma \varepsilon_{2}\right)}{\gamma^{2} \varepsilon_{2}}+2<k_{2}<\frac{2\left(\gamma^{2} y_{2}\left(k_{1}\right)-2 \gamma \varepsilon_{2}\right)}{\gamma^{2} \varepsilon_{2}}+4
\end{array}\right.
$$

Let

$$
\left\{\begin{array}{l}
k_{1}=\frac{-2\left(\gamma^{2} x_{2}(0)+2 \gamma \varepsilon_{2}\right)}{\gamma^{2} \varepsilon_{2}}+2+a_{1}, \\
k_{2}=\frac{2\left(\gamma^{2} y_{2}\left(k_{1}\right)-2 \gamma \varepsilon_{2}\right)}{\gamma^{2} \varepsilon_{2}}+2+a_{2},
\end{array}\right.
$$

where $a_{i} \in(0,2), i=1,2$. It follows from (42), (46) and (52) that

$$
\begin{aligned}
x_{2}\left(k_{1}+k_{2}+2\right) & =z_{2}\left(k_{2}\right) \\
& =y_{2}\left(k_{1}\right)+\varepsilon_{2}-k_{2} \varepsilon_{2} \\
& =x_{2}(0)+\left(k_{1}-k_{2}\right) \varepsilon_{2} \\
& =-x_{2}(0)-2 y_{2}\left(k_{1}\right)+\left(a_{1}-a_{2}\right) \varepsilon_{2} \\
& =\left(\frac{8}{\gamma}-\left(a_{1}+a_{2}+2\right)\right) \varepsilon_{2}+x_{2}(0) \\
& >10 \varepsilon_{2}+x_{2}(0)
\end{aligned}
$$

where we have noticed that $\gamma \in(0,1 / 2]$ and $a_{i} \in(0,2), i=1,2$. Therefore, from (53), we can conclude that after finite steps $k, x(k)$ in region $\mathbf{I}^{+}$satisfies $\gamma^{2} x_{2}(k) \geq 0$, namely, $x(k) \notin \Omega_{3}$, which implies that the trajectory starting at $x(0)=\left[x_{1}(0), x_{2}(0)\right]^{\mathrm{T}}$ in $\mathbf{I}^{+}$ cannot come to $\mathbf{I}^{-}$, but enter II eventually. The region $\mathbf{I}^{-}$can be considered by symmetry.

Proof of Claim 2

In region II, we have

$$
u(k)=-\sigma_{\varepsilon_{2}}\left(F_{\gamma} x(k)+\sigma_{\varepsilon_{1}}(\varpi(k))\right)=-a(k) \varepsilon_{2}, \forall k \geq 0 .
$$

where $-1 \leq a(k) \leq 1, \forall k \geq 0$. When $\left|F_{\gamma} x(k)+\sigma_{\varepsilon_{1}}(\varpi(k))\right| \leq \varepsilon_{2}$, it follows from (54) that $F_{\gamma} x(k)+\sigma_{\varepsilon_{1}}(\varpi(k))=a(k) \varepsilon_{2}$, by which we get $a(k) \varepsilon_{2}-\varepsilon_{1} \leq \gamma^{2} x_{1}(k)+2 \gamma x_{2}(k) \leq a(k) \varepsilon_{2}+\varepsilon_{1}$. When $F_{\gamma} x(k)+\sigma_{\varepsilon_{1}}(\varpi(k))>\varepsilon_{2}$, it follows from (54) that $a(k)=1$. Then $x(k)$ satisfies $a(k) \varepsilon_{2}-\varepsilon_{1} \leq \varepsilon_{2}-\sigma_{\varepsilon_{1}}(\varpi(k))<\gamma^{2} x_{1}(k)+$ $2 \gamma x_{2}(k) \leq a(k) \varepsilon_{2}+\varepsilon_{1}$, where we have noticed $F_{\gamma} x(k) \leq \varepsilon_{2}+$ $\varepsilon_{1}, \forall x(k) \in$ II. When $F_{\gamma} x(k)+\sigma_{\varepsilon_{1}}(\varpi(k))<-\varepsilon_{2}$, it follows from (54) that $a(k)=-1$. Then $x(k)$ satisfies $a(k) \varepsilon_{2}-\varepsilon_{1} \leq$ $\gamma^{2} x_{1}(k)+2 \gamma x_{2}(k)<-\varepsilon_{2}-\sigma_{\varepsilon_{1}}(\varpi(k)) \leq a(k) \varepsilon_{2}+\varepsilon_{1}$, where we have noticed $F_{\gamma} x(k) \geq-\varepsilon_{2}-\varepsilon_{1}, \forall x(k) \in$ II. In conclusion, the state $x(k)$ in region II satisfies

$$
a(k) \varepsilon_{2}-\varepsilon_{1} \leq \gamma^{2} x_{1}(k)+2 \gamma x_{2}(k) \leq a(k) \varepsilon_{2}+\varepsilon_{1}, \forall k \geq 0,
$$

where $a(k)$ is given by (54). 
Step 2.1: We first show that for any initial state $x(0) \in$ $\mathbf{I I} \backslash \Omega_{1}$, then $x(1) \in \mathbf{I}^{+}$or $\mathbf{I}^{-}$. Let us assume that $x(0)=$ $\left[x_{1}(0), x_{2}(0)\right]^{\mathrm{T}} \in \mathbf{I I}$. From (55), we have

$$
a(0) \varepsilon_{2}-\varepsilon_{1} \leq \gamma^{2} x_{1}(0)+2 \gamma x_{2}(0) \leq a(0) \varepsilon_{2}+\varepsilon_{1},
$$

where $a(0) \in[-1,1]$. Bearing in mind (54), in region II, the nonlinear system (4) can be rewritten as $x(k+1)=A x(k)-$ $b a(k) \varepsilon_{2}, \quad-1 \leq a(k) \leq 1, \forall k \geq 0$, by which we have

$$
x(1)=\left[\begin{array}{c}
x_{1}(1) \\
x_{2}(1)
\end{array}\right]=\left[\begin{array}{c}
x_{1}(0)+x_{2}(0) \\
x_{2}(0)-a(0) \varepsilon_{2}
\end{array}\right] .
$$

If $x(1) \in \mathbf{I I}$, we have

$$
-\left(\varepsilon_{1}+\varepsilon_{2}\right) \leq \gamma^{2} x_{1}(1)+2 \gamma x_{2}(1) \leq\left(\varepsilon_{1}+\varepsilon_{2}\right) .
$$

Combining (57) and (58) gives

$$
\begin{aligned}
& -\left(\varepsilon_{1}+\varepsilon_{2}\right)-\gamma^{2} x_{2}(0)+2 a(0) \gamma \varepsilon_{2} \\
\leq & \gamma^{2} x_{1}(0)+2 \gamma x_{2}(0) \\
\leq & \left(\varepsilon_{1}+\varepsilon_{2}\right)-\gamma^{2} x_{2}(0)+2 a(0) \gamma \varepsilon_{2} .
\end{aligned}
$$

Therefore, from (56) and (59) we know that, if $x(0) \in \mathbf{I I}$ and $x(1) \in$ II, then necessarily, $a(0) \varepsilon_{2}-\varepsilon_{1} \leq\left(\varepsilon_{1}+\varepsilon_{2}\right)-\gamma^{2} x_{2}(0)+2 a(0) \gamma \varepsilon_{2}$ and $a(0) \varepsilon_{2}+\varepsilon_{1} \geq-\left(\varepsilon_{1}+\varepsilon_{2}\right)-\gamma^{2} x_{2}(0)+2 a(0) \gamma \varepsilon_{2}$, namely,

$$
\begin{aligned}
-\rho_{1} & <-2 \varepsilon_{1}-\varepsilon_{2}+a(0)(2 \gamma-1) \varepsilon_{2} \\
& \leq \gamma^{2} x_{2}(0) \leq 2 \varepsilon_{1}+\varepsilon_{2}+a(0)(2 \gamma-1) \varepsilon_{2}<\rho_{1},
\end{aligned}
$$

where we have noticed $a(0) \in[-1,1]$ and $\gamma \in(0,1 / 2]$. Hence, $x(0) \in \Omega_{1}$. In conclusion, if $x(0) \in \mathbf{I I}$ and $x(1) \in \mathbf{I I}$, then necessarily $x(0) \in \Omega_{1}$. Therefore, $x(0) \in \mathbf{I I} \backslash \Omega_{1} \Rightarrow x(1) \in \mathbf{I}^{+}$or $\mathbf{I}^{-}$.

Step 2.2: We show that for any initial condition $x(0) \in \mathbf{I I} \backslash \Omega_{1}$, there exists an integer $k>0$ such that $x(k) \in \Omega_{1}$. Without loss of generality, we let $x(0) \in \mathbf{I I} \backslash \Omega_{1}, x(1) \in \mathbf{I}^{+}$. It follows that

$$
\gamma^{2} x_{2}(0)>\rho_{1} \text {. }
$$

By virtue of Claim 1, we know that the state trajectory will return to II after finite steps $k>1$, namely, $x(k)=\left[x_{1}(k), x_{2}(k)\right]^{\mathrm{T}} \in \mathbf{I I}$. If $\left|x_{2}(k)\right|-\left|x_{2}(0)\right| \leq-\kappa$ where $\kappa$ is a suitable positive constant independent of $k$, we can conclude that eventually $x$ will enter $\Omega_{1}$. Thus, it remains to show that $\left|x_{2}(k)\right|-\left|x_{2}(0)\right| \leq-\kappa$. To this end, we have the following statements, whose proofs are given in Appendix for clarity.

(1) For any initial state $x(0) \in \mathbf{I I} \backslash \Omega_{1}$ with $x(1) \in \mathbf{I}^{+}$, if the state trajectory enters II along the path II $\rightarrow \mathbf{I}^{+} \rightarrow$ II after finite steps $k$, then $\left|x_{2}(k)\right|-\left|x_{2}(0)\right| \leq-\kappa$.

(2) For any initial state $x(0) \in \mathbf{I I} \backslash \Omega_{1}$ with $x(1) \in \mathbf{I}^{+}$, if the state trajectory enters $\mathbf{I}^{-}$along the path $\mathbf{I I} \rightarrow \mathbf{I}^{+} \rightarrow \mathbf{I}^{-}$after finite steps $k$, then $\left|x_{2}(k-1)\right|-\left|x_{2}(0)\right| \leq-\kappa$.

(3) For any initial state $x(0) \in \mathbf{I}^{+}$with $x(1) \in \mathbf{I}^{-}$, if the state trajectory enters II along the path $\mathbf{I}^{+} \rightarrow \mathbf{I}^{-} \rightarrow$ II after finite steps $k$, then $\left|x_{2}(k)\right|-\left|x_{2}(0)\right| \leq-\kappa$. By symmetry, for any state $x(0) \in \mathbf{I}^{-}$with $x(1) \in \mathbf{I}^{+}$, if the state trajectory enters II along the path $\mathbf{I}^{-} \rightarrow \mathbf{I}^{+} \rightarrow$ II after finite steps $k$, the inequality $\left|x_{2}(k)\right|-\left|x_{2}(0)\right| \leq-\kappa$ holds.

(4) For any initial state $x(0) \in \mathbf{I}^{+}$with $x(1) \in \mathbf{I}^{-}$, if the state trajectory enters $\mathbf{I}^{+}$along the path $\mathbf{I}^{+} \rightarrow \mathbf{I}^{-} \rightarrow \mathbf{I}^{+}$after finite steps $k$, then $\left|x_{2}(k-1)\right|-\left|x_{2}(0)\right| \leq-\kappa$. By symmetry, for any initial state $x(0) \in \mathbf{I}^{-}$with $x(1) \in \mathbf{I}^{+}$, if the state trajectory enters $\mathbf{I}^{-}$along the path $\mathbf{I}^{-} \rightarrow \mathbf{I}^{+} \rightarrow \mathbf{I}^{-}$after finite steps $k$, then $\left|x_{2}(k-1)\right|-\left|x_{2}(0)\right| \leq-\kappa$.

From (4), we get that $x_{1}(k+1)=x_{1}(k)+x_{2}(k)$, which implies that the state trajectory moves clockwise. With the aid of the above

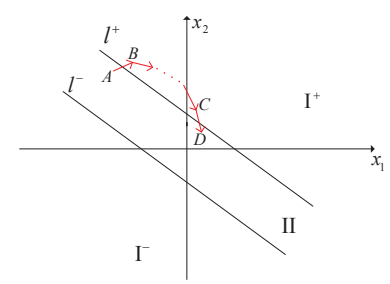

(I)

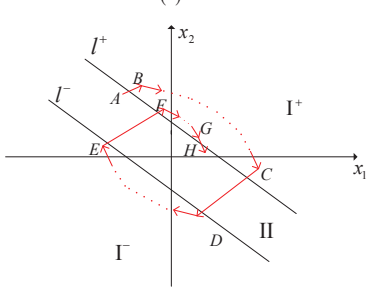

(III)

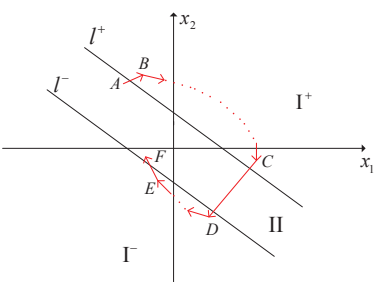

(II)

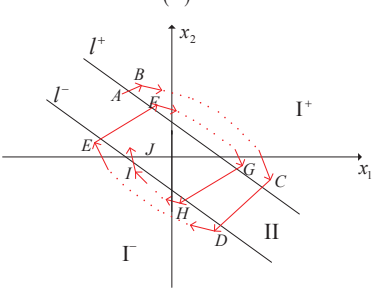

(IV)
Fig. 4. Trajectory of the planar nonlinear discrete-time system (4) under the initial condition $x(0) \in \mathbf{I I} \backslash \Omega_{1}$.

statements, and by letting $x_{\varsigma}=\left[x_{1}(\varsigma), x_{2}(\varsigma)\right]^{\mathrm{T}}$ with $\varsigma$ denoting $A, B, \ldots, J$, we have the following cases:

- II $\backslash \Omega_{1} \rightarrow \mathbf{I}^{+} \rightarrow$ II (see Fig. 4-I: $A\left(x_{A}\right) \rightarrow B\left(x_{B}\right) \rightarrow$ $C\left(x_{C}\right) \rightarrow D\left(x_{D}\right)$ ). From statement (1), we have $\left|x_{2}(D)\right|-$ $\left|x_{2}(A)\right| \leq-\kappa$.

- $\mathbf{I I} \backslash \Omega_{1} \rightarrow \mathbf{I}^{+} \rightarrow \mathbf{I}^{-} \rightarrow$ II (see Fig. 4-II: $A\left(x_{A}\right) \rightarrow \cdots \rightarrow$ $C\left(x_{C}\right) \rightarrow \cdots \rightarrow F\left(x_{F}\right)$ ). From statements (2)-(3) we have $\left|x_{2}(C)\right|-\left|x_{2}(A)\right| \leq-\kappa$ and $\left|x_{2}(F)\right|-\left|x_{2}(C)\right| \leq-\kappa$, which implies $\left|x_{2}(F)\right|-\left|x_{2}(A)\right| \leq-\kappa$.

- II $\backslash \Omega_{1} \rightarrow \mathbf{I}^{+} \rightarrow \mathbf{I}^{-} \rightarrow \mathbf{I}^{+} \rightarrow$ II (see FIG. 4-III: $A\left(x_{A}\right) \rightarrow$ $\cdots \rightarrow C\left(x_{C}\right) \rightarrow \cdots \rightarrow E\left(x_{E}\right) \rightarrow \cdots \rightarrow H\left(x_{H}\right)$ ). From statements (2)-(4) we have $\left|x_{2}(C)\right|-\left|x_{2}(A)\right| \leq-\kappa,\left|x_{2}(E)\right|-$ $\left|x_{2}(C)\right| \leq-\kappa$ and $\left|x_{2}(H)\right|-\left|x_{2}(E)\right| \leq-\kappa$, which implies $\left|x_{2}(H)\right|-\left|x_{2}(A)\right| \leq-\kappa$.

- $\mathbf{I I} \backslash \Omega_{1} \rightarrow \mathbf{I}^{+} \rightarrow \mathbf{I}^{-} \rightarrow \mathbf{I}^{+} \rightarrow \mathbf{I}^{-} \rightarrow$ II (see Fig. 4-IV: $A\left(x_{A}\right) \rightarrow \cdots \rightarrow C\left(x_{C}\right) \rightarrow \cdots \rightarrow E\left(x_{E}\right) \rightarrow \cdots \rightarrow$ $\left.G\left(x_{G}\right) \rightarrow \cdots \rightarrow J\left(x_{J}\right)\right)$. From statements (2)-(4) we have $\left|x_{2}(C)\right|-\left|x_{2}(A)\right| \leq-\kappa,\left|x_{2}(E)\right|-\left|x_{2}(C)\right| \leq-\kappa,\left|x_{2}(G)\right|-$ $\left|x_{2}(E)\right| \leq-\kappa$ and $\left|x_{2}(J)\right|-\left|x_{2}(G)\right| \leq-\kappa$, which implies $\left|x_{2}(J)\right|-\left|x_{2}(A)\right| \leq-\kappa$.

- The other cases can also be concluded from the statements (1)(4).

In conclusion, for any initial state $x(0) \in \mathbf{I I} \backslash \Omega_{1}$ with $x(1) \in$ $\mathbf{I}^{+}$, there exists an integer $k>0$ such that $x(k) \in \mathbf{I I}$ and $x_{2}(k)-$ $x_{2}(0) \leq-\kappa$, where $\kappa$ is a suitable positive constant independent of $k$. Thus, the state trajectory enters $\Omega_{1}$ after finite steps. The case with any initial condition $x(0) \in \mathbf{I I} \backslash \Omega_{1}$ with $x(1) \in \mathbf{I}^{-}$can be considered by symmetry.

\section{Proof of Claim 3}

Step 3.1: We first show that $x(0) \in \Omega_{1} \backslash \Omega_{2}, x(1) \in \mathbf{I I} \Rightarrow x(1) \in$ $\Omega_{1}$. As in the analysis of Claim 2, when $x(0) \in \Omega_{1} \backslash \Omega_{2}, x(1) \in \mathbf{I I}$, we get (57) and (60) with $a(0) \in[-1,1]$. When $a(0) \in[0,1]$, we have from (57) and (60) that $\gamma^{2} x_{2}(1)=\gamma^{2}\left(x_{2}(0)-a(0) \varepsilon_{2}\right) \leq$ $\gamma^{2} x_{2}(0)<\rho_{1}$, and $\gamma^{2} x_{2}(1)=\gamma^{2}\left(x_{2}(0)-a(0) \varepsilon_{2}\right) \geq-2 \varepsilon_{1}-$ $\varepsilon_{2}+a(0)\left(2 \gamma \varepsilon_{2}-\varepsilon_{2}-\gamma^{2} \varepsilon_{2}\right) \geq-\rho_{1}$. When $a(0) \in[-1,0)$, we have from (57) and (60) that $\gamma^{2} x_{2}(1)=\gamma^{2}\left(x_{2}(0)-a(0) \varepsilon_{2}\right) \leq$ $2 \varepsilon_{1}+\varepsilon_{2}+a(0)\left(2 \gamma \varepsilon_{2}-\varepsilon_{2}-\gamma^{2} \varepsilon_{2}\right) \leq \rho_{1}$, and $\gamma^{2} x_{2}(1)=$ $\gamma^{2}\left(x_{2}(0)-a(0) \varepsilon_{2}\right)>\gamma^{2} x_{2}(0)>-\rho_{1}$. To sum up, for any $a(0) \in[-1,1]$, we have $-\rho_{1} \leq \gamma^{2} x_{2}(1) \leq \rho_{1}$, which implies that $x(1) \in \Omega_{1}$.

Step 3.2: We show that the energy function $V(x)=x^{\mathrm{T}} P x$ is 
such that $V(x(1))-V(x(0))<0$, when $x(0) \in \Omega_{1} \backslash \Omega_{2}$ and $x(1) \in \Omega_{1}$. In region $\Omega_{1} \backslash \Omega_{2}$, we consider the following system

$$
x(k+1)=A x(k)-b \sigma_{\varepsilon_{2}}\left(F_{\gamma} x(k)+\sigma_{\varepsilon_{1}}(\varpi(k))\right) .
$$

Notice that, for any $x \in \Omega_{1} \backslash \Omega_{2}$, we have

$$
\begin{aligned}
& \sigma_{\varepsilon_{2}}\left(F_{\gamma} x(k)+\sigma_{\varepsilon_{1}}(\varpi(k))\right) \\
= & \varepsilon_{2} \sigma\left(\frac{F_{\gamma} x(k)+\sigma_{\varepsilon_{1}}(\varpi(k))}{\varepsilon_{2}}\right) \\
\epsilon & \varepsilon_{2} \operatorname{coh}\left\{\frac{F_{\gamma} x(k)+\sigma_{\varepsilon_{1}}(\varpi(k))}{\varepsilon_{2}}, \frac{F_{\gamma} x(k)}{\varepsilon_{1}+\varepsilon_{2}}\right\} \\
= & \operatorname{coh}\left\{F_{\gamma} x(k)+\sigma_{\varepsilon_{1}}(\varpi(k)), \frac{\varepsilon_{2}}{\varepsilon_{1}+\varepsilon_{2}} F_{\gamma} x(k)\right\},
\end{aligned}
$$

where $\operatorname{coh}\{\cdot\}$ denotes the convex hull [4]. Hence we only need to consider the following vertical systems of (62), namely,

$$
x(k+1)=\left(A-b F_{\gamma}\right) x(k)-b \sigma_{\varepsilon_{1}}(\varpi(k)),
$$

and

$$
x(k+1)=\left(A-\frac{\varepsilon_{2}}{\varepsilon_{1}+\varepsilon_{2}} b F_{\gamma}\right) x(k) .
$$

Denote $A_{\mathrm{c}}=A-b F_{\gamma}$. Then it is easy to verify that

$$
A_{\mathrm{c}}^{\mathrm{T}} P A_{\mathrm{c}}-P=-\gamma P-F_{\gamma}^{\mathrm{T}} F_{\gamma}, b^{\mathrm{T}} P A_{\mathrm{c}}=F_{\gamma} .
$$

It follows from (31), (32) and (65) that, the time-derivative of $V(x)=x^{\mathrm{T}} P x$ along the trajectories of system (63) satisfies

$$
\begin{aligned}
\left.\nabla V(x)\right|_{(63)}= & x^{\mathrm{T}}\left(A_{\mathrm{c}}^{\mathrm{T}} P A_{\mathrm{c}}-P\right) x-2 x^{\mathrm{T}} A_{\mathrm{c}}^{\mathrm{T}} P b \sigma_{\varepsilon_{1}}(\varpi) \\
& +\sigma_{\varepsilon_{1}}(\varpi) b^{\mathrm{T}} P b \sigma_{\varepsilon_{1}}(\varpi) \\
= & x^{\mathrm{T}}\left(-\gamma P-F_{\gamma}^{\mathrm{T}} F_{\gamma}\right) x-2 x^{\mathrm{T}} F_{\gamma}^{\mathrm{T}} \sigma_{\varepsilon_{1}}(\varpi) \\
& +\sigma_{\varepsilon_{1}}(\varpi) b^{\mathrm{T}} P b \sigma_{\varepsilon_{1}}(\varpi) \\
\leq & -\gamma x^{\mathrm{T}} P x+\left(\sigma_{\varepsilon_{1}}(\varpi)\right)^{2}+\sigma_{\varepsilon_{1}}(\varpi) b^{\mathrm{T}} P b \sigma_{\varepsilon_{1}}(\varpi) \\
< & -\gamma q+\varepsilon_{1}^{2}+\left(\frac{1}{(1-\gamma)^{2}}-1\right) \varepsilon_{1}^{2} \\
= & \left(-\gamma \mu+\frac{1}{(1-\gamma)^{2}}\right) \varepsilon_{1}^{2} \\
\triangleq & -\alpha \varepsilon_{1}^{2}, \forall x \in \Omega_{1} \backslash \Omega_{2},
\end{aligned}
$$

where $\alpha=\gamma \mu-1 /(1-\gamma)^{2}>0$. Similarly, the time-shift of $V(x)=x^{\mathrm{T}} P x$ along the trajectories of system (64) is

$$
\left.\nabla V(x)\right|_{(64)}=-x^{\mathrm{T}} R x,
$$

where

$$
\begin{aligned}
R & =P-\left(A-\frac{\varepsilon_{2}}{\varepsilon_{1}+\varepsilon_{2}} b F_{\gamma}\right)^{\mathrm{T}} P\left(A-\frac{\varepsilon_{2}}{\varepsilon_{1}+\varepsilon_{2}} b F_{\gamma}\right) \\
& =\left[\begin{array}{ll}
r_{11} & r_{12} \\
r_{21} & r_{22}
\end{array}\right]
\end{aligned}
$$

with $r_{11}=\frac{\gamma^{4} \varepsilon_{2}}{(1-\gamma)^{2}\left(\varepsilon_{1}+\varepsilon_{2}\right)^{2}}\left(\left(\gamma^{2}-2 \gamma+2\right) \varepsilon_{2}+2 \varepsilon_{1}\right), r_{22}=$ $\frac{8 \gamma^{2} \varepsilon_{2}}{(1-\gamma)^{2}\left(\varepsilon_{1}+\varepsilon_{2}\right)}+\frac{4\left(\gamma^{4}-2 \gamma^{3}\right) \varepsilon_{2}^{2}}{(1-\gamma)^{2}\left(\varepsilon_{1}+\varepsilon_{2}\right)^{2}}-\frac{\gamma^{3}+2 \gamma^{2}}{(1-\gamma)^{2}}, \quad r_{12}=r_{21}=$ $\frac{\gamma^{3}}{(1-\gamma)^{2}\left(\varepsilon_{1}+\varepsilon_{2}\right)^{2}}\left(\left(2 \gamma^{2}-4 \gamma+3\right) \varepsilon_{2}^{2}+2 \varepsilon_{1} \varepsilon_{2}-\varepsilon_{1}^{2}\right)$. One can prove easily that $\operatorname{det}(R)>\frac{\gamma^{6}}{(1-\gamma)^{4}\left(\varepsilon_{1}+\varepsilon_{2}\right)^{2}}\left(3(1-\gamma) \varepsilon_{2}-\varepsilon_{1}\right)\left((1-\gamma) \varepsilon_{2}-\varepsilon_{1}\right)$. Since $\gamma \in(0,1 / 2]$, (8) ensures that $(1-\gamma) \varepsilon_{2}-\varepsilon_{1}>0$, which implies that $R>0$. Let $\lambda_{\min }(R)$ and $\lambda_{\max }(P)$ denote respectively the minimal eigenvalue of $R$ and the maximal eigenvalue of $P$. From (67) we know that

$$
\begin{aligned}
\left.\nabla V(x)\right|_{(64)} & \leq-\lambda_{\min }(R)\|x\|^{2} \\
& =-\frac{\lambda_{\min }(R)}{\lambda_{\max }(P)}\left(\lambda_{\max }(P)\|x\|^{2}\right)
\end{aligned}
$$

$$
\begin{aligned}
& \leq-\frac{\lambda_{\min }(R)}{\lambda_{\max }(P)} V(x) \\
& <-\frac{\lambda_{\min }(R)}{\lambda_{\max }(P)} q, \forall x \in \Omega_{1} \backslash \Omega_{2},
\end{aligned}
$$

Hence, combining these two cases (66) and (68) gives $\left.\nabla V(x)\right|_{(62)}<-v_{1}<0, \quad \forall x \in \Omega_{1} \backslash \Omega_{2}$, where $v_{1} \triangleq \min \left\{\alpha, \lambda_{\min }(R) q / \lambda_{\max }(P)\right\}$, which concludes the proof of this claim.

\section{Proof of Claim 4}

Step 4.1: We first show that for any initial state $x(0) \in \Omega_{1} \backslash \Omega_{2}$, if $x(1) \in \mathbf{I}^{+}$, then $x$ will skip from $\mathbf{I}^{+}$into $\Omega_{1} \subset \mathbf{I I}$ directly after finite steps. As in the analysis of Step 1.1, the state trajectory in $\mathbf{I}^{+}$ enters $\mathbf{I I} \cup \mathbf{I}^{-}$after finite steps $k$. If $x(k) \in \mathbf{I}^{-}$, namely, the state trajectory moves along the path $\Omega_{1} \backslash \Omega_{2} \rightarrow \mathbf{I}^{+} \rightarrow \mathbf{I}^{-}$, then combining the analysis of statement (2) in Step 2.2, we have (110)-(112) and (122)-(124). It follows from (122) that

$$
\gamma^{2} y_{2}\left(k_{4}\right)<-2\left(\varepsilon_{1}+\varepsilon_{2}\right)+2 \gamma \varepsilon_{2} .
$$

Moreover, by virtue of (112) and (124), we get

$$
\begin{aligned}
\gamma^{2} y_{2}\left(k_{4}\right) & =\gamma^{2}\left(x_{2}(0)-a(0) \varepsilon_{2}-k_{4} \varepsilon_{2}\right) \\
& >\left(a(0) \gamma^{2}-2 \gamma^{2}+4 \gamma\right) \varepsilon_{2}-\gamma^{2} x_{2}(0) .
\end{aligned}
$$

Combining (69) with (70) gives $\gamma^{2} x_{2}(0)>2\left(\varepsilon_{1}+\varepsilon_{2}\right)+2 \gamma \varepsilon_{2}+$ $(a(0)-2) \gamma^{2} \varepsilon_{2}>\rho_{1}$, which is in contradiction with $x(0) \in \Omega_{1}$, where we have noticed $a(0) \in[-1,1]$ and $\gamma \in(0,1 / 2]$. Therefore, $x$ enters region II along the path $\Omega_{1} \backslash \Omega_{2} \rightarrow \mathbf{I}^{+} \rightarrow$ II after finite steps $k$. Next, we show that $x(k) \in \Omega_{1} \subset$ II. As in the analysis of statement (1) in Step 2.2, when $x$ moves along the path $\Omega_{1} \backslash \Omega_{2} \rightarrow$ $\mathbf{I}^{+} \rightarrow$ II, we have (110)-(119), and

$$
\gamma^{2} x_{2}(0) \leq \rho_{1}
$$

By (110) and (111), we have

$$
\gamma^{2} x_{2}(0)>(1+a(0)(2 \gamma-1)) \varepsilon_{2} \geq 2 \gamma \varepsilon_{2},
$$

where we have noticed $a(0) \in[-1,1]$ and $\gamma \in(0,1 / 2]$. On the one hand, it follows from (112), (119) and (71) that

$$
\begin{aligned}
\gamma^{2} x_{2}\left(k_{3}+2\right) & =\gamma^{2} y_{2}\left(k_{3}+1\right) \\
& =\gamma^{2}\left(x_{2}(0)-a(0) \varepsilon_{2}-\left(k_{3}+1\right) \varepsilon_{2}\right) \\
& =-\gamma^{2} x_{2}(0)+\gamma^{2}\left(a(0)-a_{3}-1+\frac{4}{\gamma}\right) \varepsilon_{2} \\
& \geq-2\left(\varepsilon_{1}+\varepsilon_{2}\right)+2 \gamma \varepsilon_{2}-\gamma^{2} \varepsilon_{2}-4 \gamma^{2} \varepsilon_{2}+4 \gamma \varepsilon_{2} \\
& >-2\left(\varepsilon_{1}+\varepsilon_{2}\right)+2 \gamma \varepsilon_{2}-\gamma^{2} \varepsilon_{2} \\
& =-\rho_{1},
\end{aligned}
$$

where we have noticed $a(0) \in[-1,1], a_{3} \in(0,2)$ and $\gamma \in(0,1 / 2]$. On the other hand, it follows from (112), (119) and (72) that

$$
\begin{aligned}
\gamma^{2} x_{2}\left(k_{3}+2\right) & =-\gamma^{2} x_{2}(0)+\gamma^{2}\left(a(0)-a_{3}-1+\frac{4}{\gamma}\right) \varepsilon_{2} \\
& <-2 \gamma \varepsilon_{2}+4 \gamma \varepsilon_{2} \\
& <2\left(\varepsilon_{1}+\varepsilon_{2}\right)-2 \gamma \varepsilon_{2}+\gamma^{2} \varepsilon_{2} \\
& =\rho_{1}
\end{aligned}
$$

where we have noticed $a(0) \in[-1,1], a_{3} \in(0,2)$ and $\gamma \in(0,1 / 2]$. Combining (73) and (74) gives $-\rho_{1}<\gamma^{2} x_{2}\left(k_{3}+2\right)<\rho_{1}$, which implies that $x(k) \in \Omega_{1} \subset$ II with $k=k_{3}+2$. The case with any initial condition $x(0) \in \Omega_{1} \backslash \Omega_{2}$ with $x(1) \in \mathbf{I}^{-}$can be considered by symmetry.

Step 4.2: We next show that $V(x(k))-V(x(0))<-v_{2}$ where $v_{2}$ is a positive constant independent of $k$. Based on the analysis of statement (1) in Step 2.2, we have (110)-(119). From (110), (114) 
and (117), we know that the initial state $x(0)=\left[x_{1}(0), x_{2}(0)\right]^{\mathrm{T}}$ should satisfy

$$
\left\{\begin{aligned}
f_{13} & <\gamma^{2} x_{1}(0)+2 \gamma x_{2}(0) \leq f_{17} \\
f_{15}\left(k_{3}\right) & <\gamma^{2} x_{1}(0)+2 \gamma x_{2}(0) \leq f_{16}\left(k_{3}\right)
\end{aligned}\right.
$$

where $f_{13}$ is given by (111), $f_{15}\left(k_{3}\right), f_{16}\left(k_{3}\right)$ are given by $(115)$ and $f_{17}$ is given by (116). For (75), there are four cases discussed as follows. For (75), there are four cases discussed as follows. For future use, we denote

$$
\zeta=\frac{2\left(\gamma^{2} x_{2}(0)-2 \gamma \varepsilon_{2}\right)}{\gamma^{2} \varepsilon_{2}} .
$$

Case 1: When $f_{13}<f_{15}\left(k_{3}\right), f_{16}\left(k_{3}\right)<f_{17}$, we have

$$
\left\{\begin{array}{l}
k_{3}\left(k_{3}-(\zeta+1-2 a(0))\right)>0, \\
\left(\frac{\gamma^{2} \varepsilon_{2}}{2} k_{3}+\gamma^{2} \varepsilon_{2}\right)\left(k_{3}-(\zeta+1-2 a(0))\right)<0 .
\end{array}\right.
$$

Solving (77) gives

$$
\hat{k}_{1}<k_{3}<\hat{k}_{1}
$$

where

$$
\hat{k}_{1}=\zeta+1-2 a(0),
$$

which is incompatible.

Case 2: When $f_{15}\left(k_{3}\right)<f_{13}, f_{17}<f_{16}\left(k_{3}\right)$, (78) also holds, which is incompatible.

Case 3: When $f_{13} \leq f_{15}\left(k_{3}\right)<f_{17} \leq f_{16}\left(k_{3}\right)$, we have

$$
\left\{\begin{array}{l}
k_{3}\left(k_{3}-(\zeta+1-2 a(0))\right) \geq 0, \\
\left(\frac{\gamma^{2} \varepsilon_{2}}{2} k_{3}+\frac{\gamma^{2} \varepsilon_{2}}{2}\right)\left(k_{3}-(\zeta+2-2 a(0))\right)<0 .
\end{array}\right.
$$

Solving (80) gives $\hat{k}_{1} \leq k_{3}<\hat{k}_{2}$, where $\hat{k}_{1}$ is given by (79) and

$$
\hat{k}_{2}=\zeta+2-2 a(0) .
$$

Case 4: When $f_{15}\left(k_{3}\right) \leq f_{13}<f_{16}\left(k_{3}\right) \leq f_{17}$, we have

$$
\left\{\begin{array}{l}
k_{3}\left(k_{3}-(\zeta+1-2 a(0))\right) \leq 0, \\
\left(\frac{\gamma^{2} \varepsilon_{2}}{2} k_{3}+\frac{\gamma^{2} \varepsilon_{2}}{2}\right)\left(k_{3}-(\zeta-2 a(0))\right)>0 .
\end{array}\right.
$$

Solving (82) gives $\hat{k}_{3}<k_{3} \leq \hat{k}_{1}$, where $\hat{k}_{1}$ is given by (79) and

$$
\hat{k}_{3}=\zeta-2 a(0) \text {. }
$$

To sum up, by (71)-(72) and the above four cases, we know that any initial state $x(0) \in \Omega_{1} \backslash \Omega_{2}$ generating a solution that will enter region $\mathbf{I}^{+}$and then return into $\Omega_{1}$ should satisfy

$$
\begin{aligned}
2 \gamma \varepsilon_{2} & \leq(1+a(0)(2 \gamma-1)) \varepsilon_{2} \\
& <\gamma^{2} x_{2}(0) \\
& \leq 2\left(\varepsilon_{1}+\varepsilon_{2}\right)+2 \gamma \varepsilon_{2}-3 \gamma^{2} \varepsilon_{2},
\end{aligned}
$$

and

$$
\left\{\begin{array}{cl}
f_{13}<\gamma^{2} x_{1}(0)+2 \gamma x_{2}(0) \leq f_{16}\left(k_{3}\right), & \hat{k}_{3}<k_{3} \leq \hat{k}_{1} \\
f_{15}\left(k_{3}\right)<\gamma^{2} x_{1}(0)+2 \gamma x_{2}(0) \leq f_{17}, & \hat{k}_{1} \leq k_{3}<\hat{k}_{2} .
\end{array}\right.
$$

On the one hand, from (6) and (112) we have

$$
\begin{aligned}
& V\left(x\left(k_{3}+2\right)\right)-V(x(0)) \\
= & V\left(y\left(k_{3}+1\right)\right)-V(x(0)) \\
= & \frac{2 \gamma^{2} x_{1}(0)}{(\gamma-1)^{2}} h_{1}+h_{2},
\end{aligned}
$$

where $h_{i}=h_{i}\left(k_{3}, x_{2}(0)\right), i=1,2$, and in particular, $h_{1}$ is given by

$$
h_{1}=-\frac{1}{2} \gamma \varepsilon_{2} k_{3}^{2}+\left(\left(-\frac{1}{2}-a(0)\right) \gamma \varepsilon_{2}+\gamma x_{2}(0)-\varepsilon_{2}\right) k_{3}
$$

$$
+2 \gamma x_{2}(0)-a(0) \gamma \varepsilon_{2}-(a(0)+1) \varepsilon_{2} .
$$

On the other hand, by (86), we have

$$
\begin{aligned}
& 2 \gamma x_{2}(0)-a(0) \gamma \varepsilon_{2}-(a(0)+1) \varepsilon_{2} \\
> & \frac{2 \gamma(1+a(0)(2 \gamma-1)) \varepsilon_{2}}{\gamma^{2}}-a(0) \gamma \varepsilon_{2}-(a(0)+1) \varepsilon_{2} \\
= & \frac{1}{\gamma}\left(-a(0)\left(\gamma^{2}-3 \gamma+2\right)-\gamma+2\right) \varepsilon_{2} \\
\geq & \frac{1}{\gamma}\left(2 \gamma-\gamma^{2}\right) \varepsilon_{2} \\
> & 0
\end{aligned}
$$

and

$$
\begin{aligned}
\left.h_{1}\right|_{k_{3}=\hat{k}_{2}}= & (2-\gamma) x_{2}(0)+(7-3 a(0)) \varepsilon_{2} \\
& +\left(-\frac{13}{4}+2 a(0)\right) \gamma \varepsilon_{2} \\
> & (2-\gamma) \frac{2 \gamma \varepsilon_{2}}{\gamma^{2}}+(7-3 a(0)) \varepsilon_{2} \\
& +\left(-\frac{13}{4}+2 a(0)\right) \gamma \varepsilon_{2} \\
= & -(3 a(0)+3 \gamma-2 a(0) \gamma-5) \varepsilon_{2} \\
> & 0,
\end{aligned}
$$

where we have noticed that $a(0) \in[-1,1]$ and $\gamma \in(0,1 / 2]$. Moreover, (89) can be regarded as a quadratic equation of $k_{3}$. Then it follows from (90) and (91) that $h_{1}>0, \hat{k}_{3}<k_{3}<\hat{k}_{2}$, by which and (87), (88) we have

$$
\begin{aligned}
& V\left(x\left(k_{3}+2\right)\right)-V(x(0)) \\
= & \frac{2 \gamma^{2} x_{1}(0)}{(\gamma-1)^{2}} h_{1}+h_{2} \\
\leq & \left\{\begin{array}{cl}
\frac{2\left(f_{16}\left(k_{3}\right)-2 \gamma x_{2}(0)\right)}{(\gamma-1)^{2}} h_{1}+h_{2}, & \hat{k}_{3}<k_{3} \leq \hat{k}_{1}, \\
\frac{2\left(f_{17}-2 \gamma x_{2}(0)\right)}{(\gamma-1)^{2}} h_{1}+h_{2}, & \hat{k}_{1} \leq k_{3}<\hat{k}_{2} .
\end{array}\right.
\end{aligned}
$$

Next we will give a detailed discussion about $V\left(x\left(k_{3}+2\right)\right)-V(x(0))$ based on (92).

Case 1: When $\hat{k}_{3}<k_{3} \leq \hat{k}_{1}$, we can denote $k_{3}=\hat{k}_{3}+a_{5}$ with $a_{5} \in(0,1]$, where we have noticed (79) and (83). It follows from (92) and (115) that

$V\left(x\left(k_{3}+2\right)\right)-\left.V(x(0))\right|_{k_{3}=\hat{k}_{3}+a_{5}} \leq h_{3} x_{2}^{2}(0)+h_{4} x_{2}(0)+h_{5}$,

where $h_{3}=h_{3}\left(a_{5}, \gamma\right), h_{4}=h_{4}\left(a(0), a_{5}, \gamma, \varepsilon_{1}, \varepsilon_{2}\right)$ and $h_{5}=$ $h_{5}\left(a(0), a_{5}, \gamma, \varepsilon_{1}, \varepsilon_{2}\right)$ satisfy

$$
\left\{\begin{array}{l}
h_{3}=\frac{\gamma^{2}\left(a_{5}-1\right)\left(\gamma-a_{5} \gamma+2\right)}{(\gamma-1)^{2}}, \\
h_{4}=\phi_{1} \varepsilon_{1}+\left(\phi_{2} \gamma^{3}+\phi_{3} \gamma^{2}+\phi_{4}\right) \varepsilon_{2},
\end{array}\right.
$$

with $\phi_{1}=-2 \gamma a_{5}+2 \gamma+4, \phi_{2}=-a_{5}^{3}+2 a(0) a_{5}^{2}+(1-2 a(0)) a_{5}$, $\phi_{3}=5 a_{5}^{2}-(5+6 a(0)) a_{5}+6 a(0)-2, \phi_{4}=-2 \gamma a_{5}+14 \gamma-12$. Using

$$
-1 \leq a(0) \leq 1,0<a_{5} \leq 1,0<\gamma \leq 1 / 2,
$$

we deduce from (94) and (8) that $h_{3} \leq 0$ and $h_{4}<5 \varepsilon_{1}-\frac{23}{8} \varepsilon_{2}<0$. Thus, it follows from (86) and (93) that

$$
\begin{aligned}
& V\left(x\left(k_{3}+2\right)\right)-\left.V(x(0))\right|_{k_{3}=\hat{k}_{3}+a_{5}} \\
< & h_{3}\left(\frac{2 \gamma \varepsilon_{2}}{\gamma^{2}}\right)^{2}+h_{4} \frac{2 \gamma \varepsilon_{2}}{\gamma^{2}}+h_{5} \\
= & \frac{\varepsilon_{2}}{4(\gamma-1)^{2}}\left(h_{6} \varepsilon_{1}+h_{7} \varepsilon_{2}\right),
\end{aligned}
$$

where $h_{i}=h_{i}\left(a(0), a_{5}, \gamma\right), i=6,7$, satisfy

$$
h_{6}=\phi_{5} \gamma+\phi_{6}, h_{7}=\phi_{7} \gamma^{3}+\phi_{8} \gamma^{2}+\phi_{9} \gamma+\phi_{10},
$$


with $\phi_{5}=-4 a_{5}^{2}+(8 a(0)-4) a_{5}, \phi_{6}=8 a_{5}-24 a(0)+24$, $\phi_{7}=-a_{5}^{4}+(4 a(0)-2) a_{5}^{3}+\left(-4 a^{2}(0)+4 a(0)-1\right) a_{5}^{2}, \phi_{8}=$ $(-8 a(0)+4) a_{5}^{2}+\left(16 a^{2}(0)-16 a(0)\right) a_{5}-4 a^{2}(0)+8 a(0)-4$, $\phi_{9}=12 a_{5}^{2}+(-24 a(0)+36) a_{5}-8 a^{2}(0)+8, \phi_{10}=-24 a_{5}+$ $40 a(0)-40$. In view of (95), we can compute from (97) that $h_{6}>0$. Therefore, from (96) we know that $V\left(x\left(k_{3}+2\right)\right)-V(x(0))<$ $-\frac{\gamma \varepsilon_{2}^{2}}{4(\gamma-1)^{2}}$ if $h_{6} \varepsilon_{1}+h_{7} \varepsilon_{2}<-\gamma \varepsilon_{2}$, namely,

$$
\frac{\varepsilon_{1}}{\varepsilon_{2}}<-\frac{h_{7}+\gamma}{h_{6}} .
$$

By using (95) and (97), we can compute that $-\frac{h_{7}+\gamma}{h_{6}}>0.78$, from which we know that (98) can be guaranteed by $\varepsilon_{1}<0.78 \varepsilon_{2}$, which can be further guaranteed by (8). Thus, we have

$$
V\left(x\left(k_{3}+2\right)\right)-V(x(0))<-\frac{\gamma \varepsilon_{2}^{2}}{4(\gamma-1)^{2}}, \hat{k}_{3}<k_{3} \leq \hat{k}_{1} .
$$

Case 2: When $\hat{k}_{1} \leq k_{3}<\hat{k}_{2}$, we can denote $k_{3}=\hat{k}_{3}+a_{6}$ with $a_{6} \in[0,1)$, where we have noticed (79) and (81). It follows from (92) and (116) that

$$
\begin{aligned}
& V\left(x\left(k_{3}+2\right)\right)-\left.V(x(0))\right|_{k_{3}=\hat{k}_{1}+a_{6}} \\
\leq & h_{8}\left(x_{2}(0)\right)^{2}+h_{9} x_{2}(0)+h_{10},
\end{aligned}
$$

where $h_{8}=h_{8}\left(a_{6}, \gamma\right), h_{9}=h_{9}\left(a(0), a_{6}, \gamma, \varepsilon_{1}, \varepsilon_{2}\right)$ and $h_{10}=$ $h_{10}\left(a(0), a_{6}, \gamma, \varepsilon_{1}, \varepsilon_{2}\right)$ satisfy

$$
\left\{\begin{array}{l}
h_{8}=\frac{a_{6} \gamma^{2}\left(a_{6} \gamma-2\right)}{(\gamma-1)^{2}}, \\
h_{9}=\frac{1}{(\gamma-1)^{2}}\left(\phi_{11} \varepsilon_{1}+\left(\phi_{12} \gamma^{3}+\phi_{13} \gamma^{2}+\phi_{14}\right) \varepsilon_{2}\right),
\end{array}\right.
$$

with $\phi_{11}=4-2 a_{6} \gamma, \phi_{12}=3 a_{6}^{2}+a_{6}^{3}-2 a(0) a_{6}^{2}, \phi_{13}=4 a(0) a_{6}-$ $9 a_{6}-7 a_{6}^{2}-2, \phi_{14}=12 \gamma+14 a_{6} \gamma-12$. By using

$$
-1 \leq a(0) \leq 1,0 \leq a_{6}<1,0<\gamma \leq 1 / 2,
$$

we can compute from (101) and (8) that $h_{8} \leq 0$ and $h_{9}<0$. Hence, we deduce from (86) and (100) that

$$
\begin{aligned}
& V\left(x\left(k_{3}+2\right)\right)-\left.V(x(0))\right|_{k_{3}=\hat{k}_{1}+a_{6}} \\
< & h_{8}\left(\frac{2 \gamma \varepsilon_{2}}{\gamma^{2}}\right)^{2}+h_{9} \frac{2 \gamma \varepsilon_{2}}{\gamma^{2}}+h_{10} \\
= & \frac{\varepsilon_{2}}{4(\gamma-1)^{2}}\left(h_{11} \varepsilon_{1}+h_{12} \varepsilon_{2}\right),
\end{aligned}
$$

where $h_{i}=h_{i}\left(a(0), a_{6}, \gamma\right), i=11,12$, satisfy

$$
h_{11}=\phi_{15} \gamma+\phi_{16}, h_{12}=\phi_{17} \gamma^{3}+\phi_{18} \gamma^{2}+\phi_{19} \gamma+\phi_{20} \text {, }
$$

with $\phi_{15}=-4 a_{6}^{2}+(8 a(0)-12) a_{6}+8 a(0)-8$, $\phi_{16}=8 a_{6}-24 a(0)+32, \phi_{17}=a_{6}^{4}+(6-4 a(0)) a_{6}^{3}+$ $\left(4 a^{2}(0)-12 a(0)+9\right) a_{6}^{2}-4 a^{2}(0)+8 a(0)-4, \phi_{18}=-4 a_{6}^{3}+$ $(12 a(0)-24) a_{6}^{2}+\left(-8 a^{2}(0)+36 a(0)-40\right) a_{6}+12 a^{2}(0)-$ $16 a(0), \phi_{19}=12 a_{6}^{2}+(-24 a(0)+60) a_{6}-8 a^{2}(0)-24 a(0)+56$, $\phi_{20}=-24 a_{6}+40 a(0)-64$. In view of (102), from (104) we can deduce that $h_{11}>0$. Moreover, by (103) we know that $V\left(x\left(k_{3}+2\right)\right)-V(x(0))<-\frac{\gamma \varepsilon_{2}^{2}}{4(\gamma-1)^{2}}$ if $h_{11} \varepsilon_{1}+h_{12} \varepsilon_{2}<-\gamma \varepsilon_{2}$, namely,

$$
\frac{\varepsilon_{1}}{\varepsilon_{2}}<-\frac{h_{12}+\gamma}{h_{11}} .
$$

By using (102) and (104), we can obtain $-\frac{h_{12}+\gamma}{h_{11}} \geq 1.28$, by which we know that (105) can be guaranteed by $\varepsilon_{1}<1.28 \varepsilon_{2}$, which can be further guaranteed by (8). Thus, have

$$
V\left(x\left(k_{3}+2\right)\right)-V(x(0))<-\frac{\gamma \varepsilon_{2}^{2}}{4(\gamma-1)^{2}}, \hat{k}_{1} \leq k_{3}<\hat{k}_{2} .
$$

Combining the inequalities in (99) and (106) in the above two cases gives $V\left(x\left(k_{3}+2\right)\right)-V(x(0))<-\frac{\gamma \varepsilon_{2}^{2}}{4(\gamma-1)^{2}} \triangleq-v_{2}, \hat{k}_{3}<k_{3}<\hat{k}_{2}$, where $\hat{k}_{i}, i=2,3$, are respectively given by (81) and (83). The case with any initial condition $x(0) \in \Omega_{1} \backslash \Omega_{2}$ with $x(1) \in \mathbf{I}^{-}$can be considered by symmetry. This ends the proof of the claim.

\section{Proof of Claim 5}

Step 5.1: We first show that if there exists an integer $k \geq 0$ such that $x(k) \in \Omega_{2}$, then $\left|F_{\gamma} x(k)+\sigma_{\varepsilon_{1}}(\varpi(k))\right| \leq \varepsilon_{2}$. One can check easily that

$$
F_{\gamma}^{\mathrm{T}} F_{\gamma} \leq \gamma(1-\gamma)(2-\gamma) P
$$

where $F_{\gamma}$ and $P$ are given by (5) and (6), respectively. Then, it follows from (31), (32) and (107) that

$$
\begin{aligned}
& \left|F_{\gamma} x(k)+\sigma_{\varepsilon_{1}}(\varpi)\right| \\
\leq & \left|F_{\gamma} x(k)\right|+\varepsilon_{1} \\
= & \sqrt{x^{\mathrm{T}}(k) F_{\gamma}^{\mathrm{T}} F_{\gamma} x(k)}+\varepsilon_{1} \\
\leq & \sqrt{\gamma(1-\gamma)(2-\gamma) x^{\mathrm{T}}(k) \operatorname{Px}(k)}+\varepsilon_{1} \\
\leq & \sqrt{\gamma(1-\gamma)(2-\gamma) q}+\varepsilon_{1} \\
= & (\sqrt{\gamma(1-\gamma)(2-\gamma) \mu}+1) \varepsilon_{1} \\
\leq & \varepsilon_{2} .
\end{aligned}
$$

Step 5.2: We next show that if there exists an integer $k \geq 0$ such that $x(k) \in \Omega_{2}$, then $x(k+1) \in \Omega_{2}$. It follows from (108) that in region $\Omega_{2}$, the control input $u(k)$ can be simplified as $u(k)=$ $-F_{\gamma} x(k)-\sigma_{\varepsilon_{1}}(\varpi(k))$. As a result, the system (4) can be rewritten as $x(k+1)=A_{\mathrm{c}} x(k)-b \sigma_{\varepsilon_{1}}(\varpi)$, by which and (31), (32), (65) we have

$$
\begin{aligned}
& V(x(k+1)) \\
= & x(k)^{\mathrm{T}} A_{\mathrm{c}}^{\mathrm{T}} P A_{\mathrm{c}} x(k)-2 x(k)^{\mathrm{T}} A_{\mathrm{c}}^{\mathrm{T}} P b \sigma_{\varepsilon_{1}}(\varpi(k)) \\
& +\sigma_{\varepsilon_{1}}(\varpi(k)) b^{\mathrm{T}} P b \sigma_{\varepsilon_{1}}(\varpi(k)) \\
= & (1-\gamma) V(x(k))-x(k)^{\mathrm{T}} F_{\gamma}^{\mathrm{T}} F_{\gamma} x(k) \\
& -2 x(k)^{\mathrm{T}} F_{\gamma}^{\mathrm{T}} \sigma_{\varepsilon_{1}}(\varpi(k))+\sigma_{\varepsilon_{1}}(\varpi(k)) b^{\mathrm{T}} P b \sigma_{\varepsilon_{1}}(\varpi(k)) \\
\leq & (1-\gamma) V(x(k))+\sigma_{\varepsilon_{1}}^{2}(\varpi(k)) \\
& +\sigma_{\varepsilon_{1}}(\varpi(k)) b^{\mathrm{T}} P b \sigma_{\varepsilon_{1}}(\varpi) \\
= & (1-\gamma) V(x(k))+\frac{1}{(1-\gamma)^{2}} \varepsilon_{1}^{2} \\
\leq & (1-\gamma) q+\frac{1}{(1-\gamma)^{2}} \varepsilon_{1}^{2} \\
= & q-\gamma \mu \varepsilon_{1}^{2}+\frac{1}{(1-\gamma)^{2}} \varepsilon_{1}^{2} \\
< & q-\frac{1}{\gamma(1-\gamma)^{2}} \varepsilon_{1}^{2}+\frac{1}{(1-\gamma)^{2}} \varepsilon_{1}^{2} \\
= & q,
\end{aligned}
$$

which implies that $x(k+1) \in \Omega_{2}$. This concludes the proof.

Remark 3: In view of the complexity of the proof of Claim 4, we give an example to further verify the correctness of Claim 4. Consider the following system

$$
\left\{\begin{aligned}
x(k+1) & =A x(k)+b u(k), \quad x \in \mathbf{R}^{2}, \\
u(k) & =-\sigma_{\varepsilon_{2}}\left(F_{\gamma} x(k)+\sigma_{\varepsilon_{1}}\left(-F_{\gamma} x(k)\right)\right),
\end{aligned}\right.
$$

and the energy function $V(x(k))=x^{\mathrm{T}}(k) P x(k)$, where $(A, b)$ is given by (3), $F_{\gamma}$ is given by (5), $P$ is given by (6), $\varepsilon_{i}, i=1,2$, satisfy (8), and $\gamma \in(0,1 / 2]$. For the simulations, we select $\gamma=0.4, \varepsilon_{2}=1$, $\varepsilon_{1}=0.34$ and the initial condition $x(0)=\left[x_{10}(0), x_{20}(0)\right]^{\mathrm{T}}=$ $[-45,9.5]^{\mathrm{T}}$. Then $x(0) \in \Omega_{1}$. The state trajectory and energy function are respectively plotted in Figs. 5-6. From Fig. 5, we clearly see that the state trajectory starting at $x(0)=[-45,9.5]^{\mathrm{T}} \in \Omega_{1}$ enters region $\mathbf{I}^{+}$, and then returns into $\Omega_{1}$ directly after finite steps $k=12$, which is consistent with Claim 4. Moreover, it follows from 


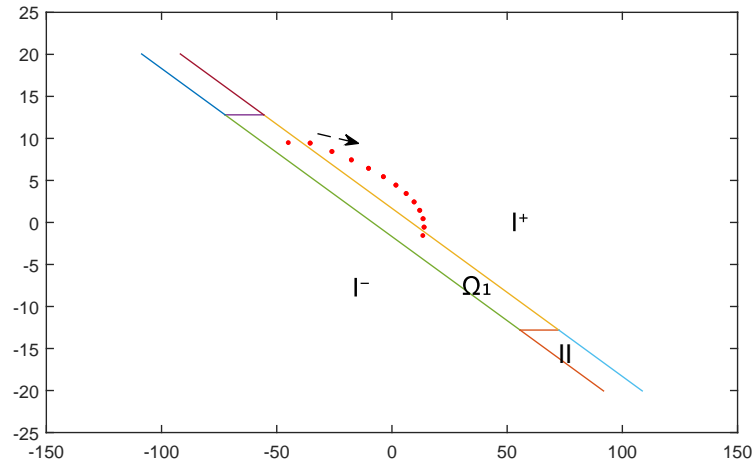

Fig. 5. The state trajectory of system (109) with $x(0)=[-45,9.5]^{\mathrm{T}}$.

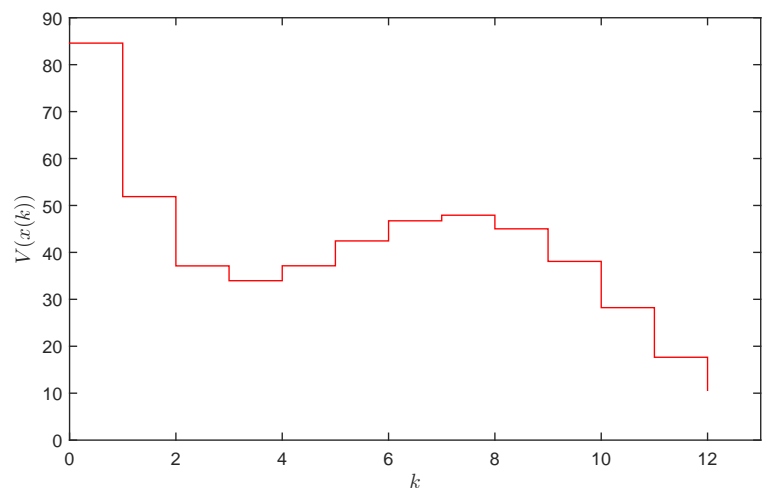

Fig. 6. The energy function $V(x(k))=x^{\mathrm{T}}(k) P x(k)$.

Fig. 6 that $V(x(12))-V(x(0))<-60$, which is also consistent with Claim 4.

\section{CONCLUSION}

In this paper, we have investigated the problem of global stabilization of discrete-time chains of integrators with length $n$ by bounded controls. Based on an energy function analysis, the solution to the global stabilization of a discrete-time double integrators system in the presence of input additive disturbances by bounded controls was given first. Then, based on a specific linear transformation, a class of nonlinear controllers consisting of only $\left[\frac{n+1}{2}\right]$ nested saturation functions were constructed for globally asymptotically stabilizing a chain of discrete-time integrators by bounded controls. The main benefit of the designed controllers over those available in the literature is that the former one needs less saturation functions, which can allow much larger control energy and can thus lead to better transient performances. This fact is cooperated by simulations we performed.

Future works will be devoted to the design of globally asymptotically stabilizing feedbacks for general ANCBC discrete-time linear systems by bounded and delayed controls and extensions of these results to nonlinear feedforward systems.

\section{APPENDIX}

Proof of statement (1) of Step 2.2 When $x(0) \in \mathbf{I I}$ and $x(1) \in$ $\mathbf{I}^{+}$, it follows from (57) that

$$
f_{13}<\gamma^{2} x_{1}(0)+2 \gamma x_{2}(0) \leq f_{14},
$$

where

$$
\left\{\begin{array}{l}
f_{13}=\left(\varepsilon_{1}+\varepsilon_{2}\right)-\gamma^{2} x_{2}(0)+2 a(0) \gamma \varepsilon_{2} \\
f_{14}=a(0) \varepsilon_{2}+\varepsilon_{1}
\end{array}\right.
$$

As in Step 1.2, in region $\mathbf{I}^{+}$we denote $y(k)=x(k+1)$. Then the state equation can be expressed as $y(k+1)=A y(k)-b \varepsilon_{2}$, with $y(0)=\left[y_{1}(0), y_{2}(0)\right]^{\mathrm{T}}=x(1)=\left[x_{1}(0)+x_{2}(0), x_{2}(0)-\right.$ $\left.a(0) \varepsilon_{2}\right]^{\mathrm{T}}$, by which, we obtain

$$
\begin{aligned}
y(k) & =A^{k}\left[\begin{array}{l}
y_{1}(0) \\
y_{2}(0)
\end{array}\right]-\sum_{i=0}^{k-1} A^{i} b \varepsilon_{2} \\
& =\left[\begin{array}{c}
x_{1}(0)+(1+k) x_{2}(0)-\frac{k(k+2 a(0)-1)}{2} \varepsilon_{2} \\
x_{2}(0)-(a(0)+k) \varepsilon_{2}
\end{array}\right] .
\end{aligned}
$$

By assumption, $y$ will enter II. Then there exists an integer $k_{3} \geq 0$ such that

$$
F_{\gamma} y\left(k_{3}\right)>\varepsilon_{1}+\varepsilon_{2}, F_{\gamma} y\left(k_{3}+1\right) \leq \varepsilon_{1}+\varepsilon_{2} .
$$

Combining (112) and (113) gives

$$
f_{15}\left(k_{3}\right)<\gamma^{2} x_{1}(0)+2 \gamma x_{2}(0) \leq f_{16}\left(k_{3}\right),
$$

with

$$
\left\{\begin{array}{l}
f_{15}\left(k_{3}\right)=\frac{1}{2} \gamma^{2} \varepsilon_{2} k_{3}^{2}+c_{1} k_{3}+c_{2}, \\
f_{16}\left(k_{3}\right)=\frac{1}{2} \gamma^{2} \varepsilon_{2} k_{3}^{2}+c_{3} k_{3}+c_{4},
\end{array}\right.
$$

where $c_{1}=\left(a(0)-\frac{1}{2}\right) \gamma^{2} \varepsilon_{2}-\gamma^{2} x_{2}(0)+2 \gamma \varepsilon_{2}, c_{2}=-\gamma^{2} x_{2}(0)+$ $(2 a(0) \gamma+1) \varepsilon_{2}+\varepsilon_{1}, c_{3}=\left(a(0)+\frac{1}{2}\right) \gamma^{2} \varepsilon_{2}-\gamma^{2} x_{2}(0)+2 \gamma \varepsilon_{2}$ and $c_{4}=-2 \gamma^{2} x_{2}(0)+\left(a(0) \gamma^{2}+2(a(0)+1) \gamma+1\right) \varepsilon_{2}+\varepsilon_{1}$. We conclude from (110) and (114) that, for any initial state $x(0) \in$ $\mathbf{I I} \backslash \Omega_{1}$ such that $x(1) \in \mathbf{I}^{+}$, if the state trajectory enters II along the path II $\rightarrow \mathbf{I}^{+} \rightarrow$ II, there must exist an integer $k_{3} \geq 0$ such that $f_{13}<f_{16}\left(k_{3}\right), f_{15}\left(k_{3}\right)<f_{14}$. Let

$$
f_{17}=\varepsilon_{1}+\varepsilon_{2}+(1-a(0)) \gamma^{2} \varepsilon_{2}-2(1-a(0)) \gamma \varepsilon_{2} .
$$

By (111) and (116) we have

$$
f_{17}-f_{14}=(1-a(0))(1-\gamma)^{2} \geq 0
$$

where we have noticed $a(0) \in[-1,1]$. Hence, $k_{3}$ satisfies the following inequalities $f_{13}<f_{16}\left(k_{3}\right), f_{15}\left(k_{3}\right)<f_{17}$, by which we have

$$
\left\{\begin{array}{l}
\left(\frac{\gamma^{2} \varepsilon_{2}}{2} k_{3}+\frac{\gamma^{2} \varepsilon_{2}}{2}\right)\left(k_{3}-(\zeta-2 a(0))\right)>0, \\
\left.\frac{\gamma^{2} \varepsilon_{2}}{2} k_{3}+\frac{\gamma^{2} \varepsilon_{2}}{2}\right)\left(k_{3}-(\zeta+2-2 a(0))\right)<0,
\end{array}\right.
$$

where $\zeta$ is given by (76). Solving (118) gives $\zeta-2 a(0)<k_{3}<$ $\zeta-2 a(0)+2$. Let

$$
k_{3}=\zeta-2 a(0)+a_{3},
$$

where $a_{3} \in(0,2)$. Then from (112) and (119) we can compute

$$
\begin{aligned}
& \left|x_{2}\left(k_{3}+2\right)\right|-\left|x_{2}(0)\right| \\
= & \left|y_{2}\left(k_{3}+1\right)\right|-\left|x_{2}(0)\right| \\
= & \left|x_{2}(0)-a(0) \varepsilon_{2}-\left(k_{3}+1\right) \varepsilon_{2}\right|-\left|x_{2}(0)\right| \\
= & \left|x_{2}(0)-\left(a(0)-a_{3}-1+\frac{4}{\gamma}\right) \varepsilon_{2}\right|-\left|x_{2}(0)\right| \\
\triangleq & \left|x_{2}(0)-\eta \varepsilon_{2}\right|-\left|x_{2}(0)\right| .
\end{aligned}
$$

Bearing in mind that $a(0) \in[-1,1], \gamma \in(0,1 / 2]$ and $a_{3} \in(0,2)$, one can check easily that $\eta=a(0)-a_{3}-1+\frac{4}{\gamma}>4>0$. In view of (61), we have $x_{2}(0)>0$. Next we consider (120). When $x_{2}(0) \geq \eta \varepsilon_{2}$, (120) gives $\left|x_{2}\left(k_{3}+2\right)\right|-\left|x_{2}(0)\right|=$ $-\eta \varepsilon_{2}<-4 \varepsilon_{2}$. When $x_{2}(0)<\eta \varepsilon_{2},(120)$ gives $\left|x_{2}\left(k_{3}+2\right)\right|-$ $\left|x_{2}(0)\right|=\frac{1}{\gamma^{2}}\left(\gamma^{2} \eta \varepsilon_{2}-2 \gamma^{2} x_{2}(0)\right)<\frac{1}{\gamma^{2}}\left(\gamma^{2} \eta \varepsilon_{2}-2 \rho_{1}\right)<$ $\frac{1}{\gamma^{2}}\left(-4 \varepsilon_{1}-2 \gamma^{2} \varepsilon_{2}\right)$, where we have noticed (61) and $a(0) \in$ $[-1,1], \gamma \in(0,1 / 2], a_{3} \in(0,2)$. To sum up, we have

$$
\left|x_{2}\left(k_{3}+2\right)\right|-\left|x_{2}(0)\right|<-\min \left\{4 \varepsilon_{2}, \frac{1}{\gamma^{2}}\left(4 \varepsilon_{1}+2 \gamma^{2} \varepsilon_{2}\right)\right\}
$$




$$
\begin{aligned}
& \leq-\min \left\{4 \varepsilon_{2}, \frac{1}{\gamma^{2}}\left(4 \varepsilon_{1}+\gamma^{2} \varepsilon_{2}\right)\right\} \\
& \triangleq-\kappa<0 .
\end{aligned}
$$

This concludes the proof of this step.

Proof of statement (2) of Step 2.2 As in the above analysis, when $x$ skips from II to $\mathbf{I}^{+}$, we have (110)-(112). When $y$ (here $y(k)=x(k+1))$ skips from $\mathbf{I}^{+}$to $\mathbf{I}^{-}$, there exists an integer $k_{4} \geq 0$ such that

$$
F_{\gamma} y\left(k_{4}\right)>\varepsilon_{1}+\varepsilon_{2}, F_{\gamma} y\left(k_{4}+1\right)<-\left(\varepsilon_{1}+\varepsilon_{2}\right) .
$$

Combining (112) and (122) gives

$$
f_{15}\left(k_{4}\right)<\gamma^{2} x_{1}(0)+2 \gamma x_{2}(0)<f_{18}\left(k_{4}\right),
$$

where $f_{18}\left(k_{4}\right)=f_{16}\left(k_{4}\right)-2\left(\varepsilon_{1}+\varepsilon_{2}\right)$, and $f_{15}\left(k_{4}\right), f_{16}\left(k_{4}\right)$ are given by (115). It follows from (110) and (123) that $k_{4}$ should satisfy $f_{13}<f_{18}\left(k_{4}\right), f_{15}\left(k_{4}\right)<f_{14}$. Since $f_{18}\left(k_{4}\right)<f_{16}\left(k_{4}\right)$ and $f_{14} \leq f_{17}$, then $k_{4}$ also satisfies the following inequalities $f_{13}<$ $f_{16}\left(k_{4}\right), f_{15}\left(k_{4}\right)<f_{17}$, by which we can deduce that

$$
\zeta-2 a(0)<k_{4}<\zeta-2 a(0)+2,
$$

where $\zeta$ is given by (76). Let

$$
k_{4}=\zeta-2 a(0)+a_{4},
$$

where $a_{4} \in(0,2)$. Then it follows from (112) that

$$
\begin{aligned}
& \left|x_{2}\left(k_{4}+1\right)\right|-\left|x_{2}(0)\right| \\
= & \left|y_{2}\left(k_{4}\right)\right|-\left|x_{2}(0)\right| \\
= & \left|x_{2}(0)-a(0) \varepsilon_{2}-k_{4} \varepsilon_{2}\right|-\left|x_{2}(0)\right| \\
= & \left|x_{2}(0)-\left(a(0)-a_{4}+\frac{4}{\gamma}\right) \varepsilon_{2}\right|-\left|x_{2}(0)\right| .
\end{aligned}
$$

Since $a(0) \in[-1,1], \gamma \in(0,1 / 2]$ and $a_{4} \in(0,2)$, one can easily verify that $a(0)-a_{4}+\frac{4}{\gamma}>5>0$. Then, as in the proof of statement (1), we conclude from $a(0) \in[-1,1], \gamma \in(0,1 / 2], a_{4} \in(0,2)$ and (61) that (126) can be reduced to $\left|x_{2}\left(k_{4}+1\right)\right|-\left|x_{2}(0)\right|<$ $-\min \left\{5 \varepsilon_{2},\left(4 \varepsilon_{1}+\gamma^{2} \varepsilon_{2}\right) / \gamma^{2}\right\} \leq-\kappa$, where $\kappa$ is given by $(121)$, which ends the proof.

Proof of statement (3) of Step 2.2 As in the analysis of Step 1.2 , when $x$ skips from $\mathbf{I}^{+}$to $\mathbf{I}^{-}$, we have (39)-(42). When $y$ (here $y(k)=x(k+1))$ skips from $\mathbf{I}^{-}$to $\mathbf{I I}$, there exists an integer $k_{5} \geq 0$ such that

$$
F_{\gamma} y\left(k_{5}\right)<-\left(\varepsilon_{1}+\varepsilon_{2}\right), F_{\gamma} y\left(k_{5}+1\right) \geq-\left(\varepsilon_{1}+\varepsilon_{2}\right) .
$$

Combining (42) and (127) gives

$$
f_{19}\left(k_{5}\right) \leq \gamma^{2} x_{1}(0)+2 \gamma x_{2}(0)<f_{20}\left(k_{5}\right),
$$

where

$$
\left\{\begin{aligned}
f_{19}\left(k_{5}\right)= & -\frac{1}{2} \gamma^{2} \varepsilon_{2} k_{5}^{2}+\left(\frac{1}{2} \gamma^{2} \varepsilon_{2}-\gamma^{2} x_{2}(0)-2 \gamma \varepsilon_{2}\right) k_{5} \\
& -2 \gamma^{2} x_{2}(0)+\gamma^{2} \varepsilon_{2}-\left(\varepsilon_{1}+\varepsilon_{2}\right), \\
f_{20}\left(k_{5}\right)= & -\frac{1}{2} \gamma^{2} \varepsilon_{2} k_{5}^{2}+\left(\frac{3}{2} \gamma^{2} \varepsilon_{2}-\gamma^{2} x_{2}(0)-2 \gamma \varepsilon_{2}\right) k_{5} \\
& -\gamma^{2} x_{2}(0)+2 \gamma \varepsilon_{2}-\left(\varepsilon_{1}+\varepsilon_{2}\right) .
\end{aligned}\right.
$$

It follows from (39) and (128) that $k_{5}$ should satisfy $f_{1}<$ $f_{20}\left(k_{5}\right), f_{19}\left(k_{5}\right)<f_{2}$. Since $f_{1}>f_{9}$ (see (50)), $k_{5}$ also satisfies the following inequalities $f_{9}<f_{20}\left(k_{5}\right), f_{19}\left(k_{5}\right)<f_{2}$, by which we get

$$
k_{5}=\frac{-2\left(\gamma^{2} x_{2}(0)+2 \gamma \varepsilon_{2}\right)}{\gamma^{2} \varepsilon_{2}}+2+a_{5},
$$

where $a_{5} \in(0,2)$. By virtue of (42) and (130), we have

$$
\begin{aligned}
& \left|x_{2}\left(k_{5}+2\right)\right|-\left|x_{2}(0)\right| \\
& =\left|y_{2}\left(k_{5}+1\right)\right|-|| x_{2}(0)||
\end{aligned}
$$

$$
\begin{aligned}
& =\left|x_{2}(0)-\varepsilon_{2}+\left(k_{5}+1\right) \varepsilon_{2}\right|-\left|y_{2}\left(k_{4}\right)\right| \\
& =\left|x_{2}(0)-\left(-\frac{4}{\gamma}+2+a_{5}\right) \varepsilon_{2}\right|-\left|x_{2}(0)\right| \\
& \triangleq\left|x_{2}(0)-\xi \varepsilon_{2}\right|-\left|x_{2}(0)\right| .
\end{aligned}
$$

Notice that, by using $\gamma \in(0,1 / 2]$ and $a_{4} \in(0,2)$, one can check easily that $\xi=-\frac{4}{\gamma}+2+a_{5}<-4<0$. In view of (41), we have $x_{2}(0)>0$. Next we consider (131). When $x_{2}(0) \geq \xi \varepsilon_{2}$, (131) can be continued as $\left|x_{2}\left(k_{5}+2\right)\right|-\left|x_{2}(0)\right|=\left(2 \gamma^{2} x_{2}(0)-\gamma^{2} \xi \varepsilon_{2}\right) / \gamma^{2}<$ $\left(-4 \varepsilon_{1}+(-4+8 \gamma) \varepsilon_{2}-\left(2+a_{5}\right) \gamma^{2} \varepsilon_{2}\right) / \gamma^{2}<\left(-4 \varepsilon_{1}-2 \gamma^{2} \varepsilon_{2}\right) / \gamma^{2}$. When $x_{2}(0)<\xi \varepsilon_{2}$, (131) gives $\left|x_{2}\left(k_{5}+2\right)\right|-\left|x_{2}(0)\right|=$ $\xi \varepsilon_{2}<-4 \varepsilon_{2}$. To sum up, we have $\left|x_{2}\left(k_{5}+2\right)\right|-\left|x_{2}(0)\right|<$ $-\min \left\{4 \varepsilon_{2}, \frac{1}{\gamma^{2}}\left(4 \varepsilon_{1}+2 \gamma^{2} \varepsilon_{2}\right)\right\} \leq-\kappa$, where $\kappa$ is given by $(121)$, which ends the proof of this step.

Proof of statement (4) of Step 2.2 As in the analysis of Step 1.2 , when $x$ skips from $\mathbf{I}^{+}$to $\mathbf{I}^{-}$and then returns to $\mathbf{I}^{+}$, we have (38)-(45) with $k_{1}$ satisfying (52). By virtue of (42) and (52), we get

$$
\begin{aligned}
& \left|x_{2}\left(k_{1}+1\right)\right|-\left|x_{2}(0)\right| \\
= & \left|y_{2}\left(k_{1}\right)\right|-\left|x_{2}(0)\right| \\
= & \left|x_{2}(0)-\varepsilon_{2}+k_{1} \varepsilon_{2}\right|-\left|x_{2}(0)\right| \\
= & \left|x_{2}(0)-\left(-\frac{4}{\gamma}+1+a_{1}\right) \varepsilon_{2}\right|-\left|x_{2}(0)\right| .
\end{aligned}
$$

Since $\gamma \in(0,1 / 2]$ and $a_{1} \in(0,2)$, one can check easily that $-\frac{4}{\gamma}+$ $1+a_{1}<-5<0$. As in the proof of statement (3), we conclude from $\gamma \in(0,1 / 2], a_{1} \in(0,2)$ and (41) that (132) can be continued as $\left|x\left(k_{1}+1\right)\right|-\left|x_{2}(0)\right|<-\min \left\{5 \varepsilon_{2},\left(4 \varepsilon_{1}+\gamma^{2} \varepsilon_{2}\right) / \gamma^{2}\right\} \leq-\kappa$, where $\kappa$ is given by (121). This completes the proof.

\section{REFERENCES}

[1] S. Amini, B. Ahi, and M. Haeri, "Control of high order integrator chain systems subjected to disturbance and saturated control: A new adaptive scheme," Automatica, vol. 100, pp. 108-113, 2019.

[2] H. Fang and Z. Lin, "A further result on global stabilization of oscillators with bounded delayed input," IEEE Transactions on Automatic Control, vol. 51, pp. 121-128, 2006.

[3] A. Fuller, "In-the-large stability of relay and saturating control systems with linear controllers," International Journal of Control, vol. 10, pp. 457-480, 1969.

[4] T. Hu and Z. Lin, Control systems with actuator saturation: analysis and design. Secaucus, USA: Birkhäuser, 2001.

[5] B. Jiang, J. Lu, Y. Liu, and J. Cao, "Periodic event-triggered adaptive control for attitude stabilization under input saturation," IEEE Transactions on Circuits and Systems I: Regular Papers, vol. 67, pp. 249-258, 2020.

[6] G. Kaliora and A. Astolfi,"Nonlinear control of feedforward systems with bounded signals," IEEE Transanctions on Automatic Control, vol. 49, pp. 1975-1990, 2004.

[7] J. Laporte, A. Chaillet, and Y. Chitour, "Global stabilization of classes of linear control systems with bounds on the feedback and its successive derivatives, " Systems \& Control Letters, vol. 99, pp. 17-24, 2017.

[8] Y. Lv, J. Fu, G. Wen, T. Huang, and X. Yu,"Distributed adaptive observer-based control for output consensus of heterogeneous MASs with input saturation constraint, "IEEE Transactions on Circuits and Systems I: Regular Papers, doi: 10.1109/TCSI.2019.2955163.

[9] R. Lozano and D. Dimogianopoulos, "Stabilization of a chain of integrators with nonlinear perturbations: applications to the inverted pendulum," Proceedings of the 42nd IEEE Conference on Decision and Control, pp. 5191-5196, 2003

[10] N. Marchand, "Further results on global stabilization for multiple integrators with bounded controls," Proceedings of the 42nd IEEE Conference on Decision and Control, pp. 4440-4444, 2003.

[11] N. Marchand and A. Hably,"Global stabilization of multiple integrators with bounded controls, " Automatica, vol. 41, pp. 2147-2152, 2005.

[12] N. Marchand, A. Hably, and A. Chemori,"Global stabilization with low computational cost of the discrete-time chain of integrators by means of bounded controls," IEEE Transanctions on Automatic Control, vol. 52, pp. 948-952, 2007. 
[13] F. Mazenc, S. Mondié, and S.-I. Niculescu, "Global asymptotic stabilization for chains of integrators with a delay in the input," IEEE Transanctions on Automatic Control, vol. 48, pp. 57-63, 2003.

[14] W. Qi, G. Zong, and H. Karim,"'Observer-based adaptive SMC for nonlinear uncertain singular semi-Markov jump systems with applications to DC motor," IEEE Transactions on Circuits and Systems I: Regular Papers, vol. 65, pp. 2951-2960, 2018.

[15] G. Shi, A. Saberi, "On the input-to-state stability (ISS) of a double integrator with saturated linear control laws," Proceedings of American Control Conference, pp. 59-61, 2002.

[16] H. J. Sussmann, E. D. Sontag, and Y. Yang,"A general result on the stabilization of linear systems using bounded controls," IEEE Transanctions on Automatic Control, vol. 39, pp. 2411-2425, 1994.

[17] H. J. Sussmann and Y. Yang, "On the stabilizability of multiple integrators by means of bounded feedback controls," Proceedings of the 30nd IEEE Conference on Decision and Control, pp. 70-72, 1991.

[18] A. R. Teel, "Global stabilization and restricted tracking for multiple integrators with bounded controls," Systems \& Control Letters, vol. 18, pp. 165-171, 1992.

[19] F. Tyan and D. S. Bernstein, "Global stabilization of systems containing a double integrator using a saturated linear controller," International Journal of Robust and Nonlinear Control, vol. 9, pp. 1143-1156, 1999.

[20] Y. Wei, H. Yu, H. Karimi, and Y. Joo,"New approach to fixedorder output-feedback control for piecewise-affine systems," IEEE Transactions on Circuits and Systems I: Regular Papers, vol. 65, pp. 2961-2969, 2018.

[21] T. Yang, A. A. Stoorvogel, and A. Saberi,"Global stabilization of the discrete-time double integrator using a saturated linear state feedback controller," Proceedings of American Control Conference (ACC), pp. 4440-4445, 2011.

[22] T. Yang, A. A. Stoorvogel, X. Wang, and A. Saberi, "Periodic behavior of locally stabilizing saturated linear controllers for the discrete-time double integrator," IFAC Proceedings Volumes, vol. 43, pp. 237-241, 2010.

[23] Y. D. Yang, E. D. Sontag, and H. J. Sussmann, "Global stabilization of linear discrete-time systems with bounded feedback," Systems \& Control Letters, vol. 30, pp. 273-281, 1997.

[24] H. Ye, H. Wang and H. Wang,"Stabilization of a PVTOL aircraft and an inertia wheel pendulum using saturation technique," IEEE Transactions on Control Systems Technology, vol. 15, pp. 1143-1150, 2007.

[25] A. Zavala-Río, I. Fantoni, and R. Lozano,"Global stabilization of a PVTOL aircraft model with bounded inputs," International Journal of Control, vol. 76, pp. 1833-1844, 2003.

[26] B. Zhou and G. R. Duan, "Global stabilization of linear systems via bounded controls," Systems \& Control Letters, vol.58, pp. 54-61, 2009.

[27] B. Zhou, G. Duan, and Z. Li, “On improving transient performance in global control of multiple integrators system by bounded feedback," Systems \& Control Letters, Vol. 57, pp. 867-875, 2008.

[28] B. Zhou and Y. Liu,"Global stabilization of linear systems with bounded controls by the energy function with applications to the spacecraft rendezvous," Proceedings of 2017 Chinese Automation Congress, pp. 2428-2435, 2017.

[29] B. Zhou and X. Yang, "Global stabilization of the multiple integrators system by delayed and bounded controls,"IEEE Transactions on Automatic Control, vol. 61, pp. 4222-4228, 2016.

[30] B. Zhou and X. Yang,"Global stabilization of discrete-time multiple integrators with bounded and delayed feedback," Automatica, vol. 97, pp. 306-315, 2018.

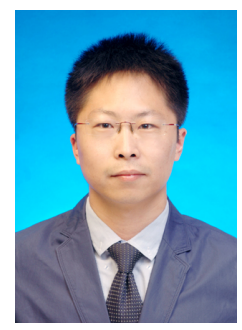

Xuefei Yang was born in 1989. He received the B.S. degree from the Department of Mathematics, the M.S. degree from the department of Materials Processing Engineering, the Ph.D. degree from the Department of Control Science and Engineering at Harbin Institute of Technology, Harbin, China in 2012, 2014 and 2018, respectively. He received the "National Postdoctoral Program for Innovative Talents" in 2019. He is currently a Postdoctor in the Department of Mechanical Engineering \& Automation, Harbin Institute of Technology(Shenzhen). His current research interests include nonlinear control systems, time-delay systems.

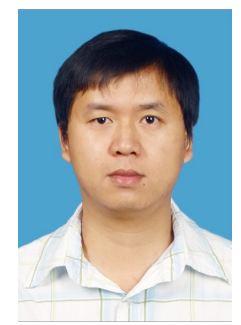

Bin Zhou (SM'16) is a Professor of the Department of Control Science and Engineering at the Harbin Institute of Technology. He received the Bachelors degree, the Masters Degree and the Ph.D. degree from the Department of Control Science and Engineering at Harbin Institute of Technology, Harbin, China in 2004, 2006 and 2010, respectively. His current research interests include constrained control, time-delay systems, time-varying systems, nonlinear control, multi-agent systems, and control applications in astronautic engineering. In these areas, he has published over 100 papers in archival journals. He is the author of the book Truncated Predictor Feedback for Time-Delay Systems (Springer-Verlag, Berlin Heidelberg, 2014). He is a reviewer for American Mathematical Review and is an active reviewer for many journals. He received the National Excellent Doctoral Dissertation Award in 2012 from the Academic Degrees Committee of the State Council and the Ministry of Education of P.R. China. He is currently an associate editor on the Conference Editorial Board of the IEEE Control Systems Society and an Associate Editor of IET Control Theory \& Applications, Asian Journal of Control, Journal of System Science and Mathematical Science and Control and Decision.

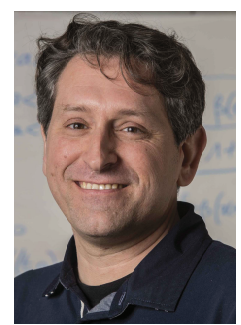

Frdric Mazenc received his Ph.D. in Automatic Control and Mathematics from the CAS at Ecole des Mines de Paris in 1996. He was a Postdoctoral Fellow at CESAME at the University of Louvain in 1997. From 1998 to 1999, he was a Postdoctoral Fellow at the Centre for Process Systems Engineering at Imperial College. He was a CR at INRIA Lorraine from October 1999 to January 2004. From 2004 to 2009, he was a CR1 at INRIA SophiaAntipolis. From 2010, he was a CR1 at INRIA Saclay. He received a best paper award from the IEEE Transactions on Control Systems Technology at the 2006 IEEE Conference on Decision and Control. His current research interests include nonlinear control theory, differential equations with delay, robust control, and microbial ecology. He has more than 200 peer reviewed publications. Together with Michael Malisoff, he authored a research monograph entitled Constructions of Strict Lyapunov Functions in the Springer Communications and Control Engineering Series. 